\title{
Nutritional Status and Nutritional Treatment Are Related to Outcomes and Mortality in Older Adults with Hip Fracture
}

\author{
Vincenzo Malafarina ${ }^{1,2, *(1)}$, Jean-Yves Reginster ${ }^{3,4,5}$, Sonia Cabrerizo ${ }^{6}$, Olivier Bruyère ${ }^{3,4}$, \\ John A. Kanis ${ }^{7,8}$, J. Alfredo Martinez 1,9,10,11,12 (iD) and M. Angeles Zulet 1,9,10,11 \\ 1 Department of Nutrition, Food Science and Physiology, Faculty of Pharmacy and Nutrition, \\ University of Navarra, 31008 Pamplona, Spain; jalfmtz@unav.es (J.A.M.); mazulet@unav.es (M.A.Z.) \\ 2 Geriatric Department, Complejo Hospitalario de Navarra, 31008 Pamplona, Spain \\ 3 Department of Public Health, Epidemiology and Health Economics, University of Liège, CHUB23, 4000, \\ Liège, Belgium; jyr.ch@bluewin.ch (J.-Y.R.); olivier.bruyere@ulg.ac.be (O.B.) \\ 4 WHO Collaborating Center for Public Health Aspects of Musculoskeletal Health and Aging, Liège, Belgium \\ 5 King Saud University, 11692 Riyadh, Kingdom of Saudi Arabia \\ 6 Dietician Researcher; 08025 Barcelona, Spain, sonia.abril51@gmail.com \\ 7 Centre for Metabolic Bone Diseases, University of Sheffield Medical School, University of Sheffield, \\ Beech Hill Road, Sheffield S10 2RX, UK; w.j.pontefract@sheffield.ac.uk \\ 8 Institute for Health and Aging, Catholic University of Australia, Fitzroy VIC 3065 Melbourne, Australia \\ 9 Navarra Institute for Health Research (IdiSNA), 31008, Pamplona, Spain \\ 10 CIBERobn, Physiopathology of Obesity and Nutrition, Instituto de Salud Carlos III, 28029 Madrid, Spain \\ 11 Centre for Nutrition Research, Faculty of Pharmacy and Nutrition, University of Navarra, \\ 31008 Pamplona, Spain \\ 12 IMDEA Food, Research Institute on Food \& Health Sciences, 28049 Madrid, Spain. \\ * Correspondence: vmalafarina@gmail.com; Tel.: +34-94-842-5600 (ext. 6317); Fax: +34-94-842-5740
}

Received: 31 January 2018; Accepted: 25 April 2018; Published: 30 April 2018

\begin{abstract}
Malnutrition is very prevalent in geriatric patients with hip fracture. Nevertheless, its importance is not fully recognized. The objective of this paper is to review the impact of malnutrition and of nutritional treatment upon outcomes and mortality in older people with hip fracture. We searched the PubMed database for studies evaluating nutritional aspects in people aged 70 years and over with hip fracture. The total number of studies included in the review was 44 , which analyzed 26,281 subjects $(73.5 \%$ women, $83.6 \pm 7.2$ years old). Older people with hip fracture presented an inadequate nutrient intake for their requirements, which caused deterioration in their already compromised nutritional status. The prevalence of malnutrition was approximately $18.7 \%$ using the Mini-Nutritional Assessment (MNA) (large or short form) as a diagnostic tool, but the prevalence was greater (45.7\%) if different criteria were used (such as Body Mass Index (BMI), weight loss, or albumin concentration). Low scores in anthropometric indices were associated with a higher prevalence of complications during hospitalization and with a worse functional recovery. Despite improvements in the treatment of geriatric patients with hip fracture, mortality was still unacceptably high (30\% within 1 year and up to $40 \%$ within 3 years). Malnutrition was associated with an increase in mortality. Nutritional intervention was cost effective and was associated with an improvement in nutritional status and a greater functional recovery. To conclude, in older people, the prevention of malnutrition and an early nutritional intervention can improve recovery following a hip fracture.
\end{abstract}

Keywords: older adults; hip fracture; malnutrition; body mass index; nutritional biomarkers 


\section{Introduction}

Hip fractures represent a significant health risk for older populations because the incidence of fractures increases notably with age [1].

Hip fractures in geriatric patients have a negative impact on functional status and quality of life, and are associated with high mortality [2,3]. Despite the reduction in pre-surgery hospital stay (surgery performed in the first $24 \mathrm{~h}$, or $48 \mathrm{~h}$ after admission, is associated with fewer post-operative complications) [4], and improvements in the management of complications, many patients with hip fracture presented functional deterioration [5]. Identifying the risk factors that predict functional loss after a hip fracture could reduce the costs associated with the need for help resulting from loss of autonomy [6] and institutionalization [7], and could also improve the treatment of post-operative complications. The need for help in order to be able to walk within a patient's home, Parkinson's disease, smoking, having suffered delirium in the previous month, having a Body Mass Index (BMI) $<22 \mathrm{~kg} / \mathrm{m}^{2}$, and age are among the independent risk factors for hip fractures [8]. Poor nutritional status, defined by the Mini Nutritional Assessment (MNA), was associated with a higher risk of fracture at any site [9]. Among risk factors for hip fracture as well as functional loss after the fracture, malnutrition represents an area of great interest, principally because it is a modifiable risk factor. The identification of malnutrition is widely accepted as an appropriate procedure, which may help to give patients better care [10]. This review represents an actualization of the evidence previously published on this topic. The novelty of this review is that we included not only studies with nutritional interventions, but also studies that have assessed the nutritional status in older patients with hip fracture.

The principal objective of this review is to describe how both nutritional status, as revealed by malnutrition biomarkers, influences the clinical evolution and mortality of older people with hip fracture, as well as the impact of nutritional intervention. We therefore structured this paper into four chapters concerning subjects with hip fracture: (1) prevalence of malnutrition and nutritional status aspects (including anthropometry, blood biomarkers, and energy intake), (2) influence upon outcomes and complications, (3) mortality, and (4) effects of nutritional intervention.

\section{Material and Methods}

\subsection{Data Sources and Search Strategy}

A search was carried out on the electronic database MEDLINE for papers published from January 1990 until December 2017. The search strategy is detailed in Supplementary data. The search was restricted to articles in English, Spanish, or Italian. The references of the selected articles were manually revised in the search for eligible articles. Whenever there were studies with multiple publications about the same population, the study with the largest sample was selected, as long as it respected our inclusion criteria.

\subsection{Inclusion and Exclusion Criteria}

We included observational and cohort studies that evaluated the presence of malnutrition (defined by MNA, BMI, albumin concentration, or weight loss), and the influence of malnutrition, as revealed by nutritional biomarkers, on functional recovery, post-operative complications, and mortality in hip fracture patients. We considered as nutritional biomarkers: (1) anthropometric parameters, such as BMI, mid-arm circumference, and triceps skinfold; (2) blood concentrations of total proteins, albumin, and micronutrients such as vitamin D and calcium. We also included controlled clinical trials with nutritional intervention. We defined an intervention as cases where patients received supplements (either orally, by tube, or intravenously) or advice on the characteristics of the diet (by a specialized nurse or dietician). We consider studies (which included only males, only females, or both sexes) carried out in populations with an average age of 70 years or above. Reviews and protocols that did not provide results were excluded. 


\subsection{Data Extraction}

The title and abstract of papers compiled from the search were evaluated by two researchers who carried out data extraction. Doubts and queries were discussed and whenever these could not be solved, the opinion of a third reviewer was requested. Studies were grouped according to their main objective. When necessary we contacted the corresponding author to request data that did not appear in the paper.

\subsection{Quality Assessment}

The quality of the selected studies was determined with both the National Institutes of Health (NIH) Quality Assessment tool for Observational Cohort and Cross-Sectional Studies and the Quality Assessment of Controlled Intervention Studies [11]. These tools have been designed to evaluate internal validity and bias risk for both types of observational and intervention studies, and each consists of 14 evaluation criteria. The criteria for observational studies are: aims of the study, sources of bias, sampling, participation rate, study power, data collection methods, and confounding. The criteria for intervention studies are: objective of the study, population characteristics, sampling, selection criteria, sample size justification, exposure measured, timeframe, categories of exposure, independent variables, exposure over the time, dependent variables, blinded, drop-out, and confounding. The criteria were rated as either yes, no, or "other" (i.e., CD, cannot determine; NA, not applicable; NR, not reported). The overall assessment of the studies were classified as "good", "fair", or "poor".

\section{Results}

This review included 44 papers, which totaled 26,281 subjects with a mean age $83.6 \pm 7.2$ years. The population was mostly female (73.5\%). The overall quality of the included studies was rated as fair (Supplementary Tables S1 and S2).

\subsection{Prevalence of Malnutrition and Nutritional Status Aspects in Hip Fracture Patients}

In all of the studies included, malnutrition was identified by a validated nutritional assessment tool. Nevertheless, the prevalence of malnutrition changed according to diagnostic tool used. The prevalence of malnutrition was $18.7 \%$ using the MNA (long or short form), but it was greater if other diagnostic criteria were used (BMI, albumin, or weight loss) $(45.7 \%)$. The prevalence of malnutrition, of risk of malnutrition, and the diagnostic tool used in each study are presented in Table 1.

In this section we included 10 studies that assessed the nutritional status of older people with hip fracture, with a total of 1575 subjects ( $88.3 \%$ female, mean age $79.6 \pm 4$ years). The design of the studies, the general characteristics of the populations studied, and the main results are presented in Table 2.

Patients with hip fracture present malnutrition, as demonstrated by the presence of low values of the anthropometric indices. Several studies showed that energy intake in older people is smaller than that required and recommended [12-15]. They also showed that calorie and protein intake are significantly lower in geriatric patients with hip fracture compared to patients without fracture. Both the reduced intake observed in hip fracture patients and the increase of the energy requirement secondary to the inflammatory state lead to weight loss and a reduction in muscle mass and fat tissue indicators, and this hypercatabolism situation may continue up to 4 months after the fracture [16-18].

The importance of a good nutritional status was backed up by studies that observed how higher BMI scores were associated with a lower incidence of hip fractures [19]. An interesting and original study showed that patients with intracapsular fractures presented lower BMI scores than patients with intertrochanteric fractures. Almost half of the subjects with intracapsular fractures presented BMI scores lower than $18 \mathrm{~kg} / \mathrm{m}^{2}$, versus only one-fifth of patients with intertrochanteric fractures [20]. 
Table 1. Prevalence of malnutrition or risk of malnutrition and nutritional screening tool used in the included studies.

\begin{tabular}{|c|c|c|c|c|c|}
\hline Reference & $\begin{array}{c}\text { Total } \\
n\end{array}$ & $\begin{array}{c}\mathrm{WN} \\
n\end{array}$ & $\underset{n}{\mathrm{RMN}}$ & $\begin{array}{c}\mathrm{MN} \\
n\end{array}$ & Cut-Off for Malnutrition \\
\hline [21] & 17,651 & 9549 & - & 8102 & Albumin $<3.5 \mathrm{~g} / \mathrm{dL}$ \\
\hline [22] & 173 & 49 & - & 57 & $\mathrm{BMI}<22 \mathrm{~kg} / \mathrm{m}^{2}$ \\
\hline [23] & 23 & 9 & 7 & 7 & $\mathrm{BMI}^{+}$ \\
\hline [20] & 96 & 59 & - & 37 & $\mathrm{BMI}<18.5 \mathrm{~kg} / \mathrm{m}^{2}$ \\
\hline$[24]$ & 60 & 34 & - & 26 & $\begin{array}{c}\text { Weight loss } \geq 5 \% 1 \mathrm{~m}, \text { or } \geq 10 \% 6 \mathrm{~m}, \\
\text { and / or albumin }<2.7 \mathrm{~g} / \mathrm{dL}\end{array}$ \\
\hline [14] & 25 & 11 & 11 & 3 & Hospital's own screening tool $\S$ \\
\hline $\begin{array}{l}\text { Total of } \\
\text { subjects }\end{array}$ & 18,028 & 9711 & 18 & 8232 & \\
\hline Percentage & & $53.9 \%$ & & $45.7 \%$ & \\
\hline Reference & $\begin{array}{c}\text { Total } \\
n\end{array}$ & $\begin{array}{c}\mathrm{WN} \\
n\end{array}$ & $\begin{array}{c}\mathrm{RMN} \\
n\end{array}$ & $\begin{array}{c}\mathrm{MN} \\
n\end{array}$ & Cut-Off for Malnutrition \\
\hline [15] & 49 & 18 & 23 & 8 & MNA $\ddagger$ \\
\hline [19] & 80 & 38 & 35 & 7 & MNA \\
\hline [25] & 127 & 89 & 36 & 2 & MNA \\
\hline [17] & 50 & 32 & 18 & 0 & MNA \\
\hline [26] & 50 & 7 & 29 & 14 & MNA \\
\hline [27] & 97 & 44 & 37 & 16 & MNA \\
\hline [28] & 162 & 59 & - & 103 & MNA \\
\hline [29] & 152 & 87 & - & 65 & MNA \\
\hline [18] & 215 & 95 & 95 & 25 & MNA-SF $¥$ \\
\hline [30] & 204 & 55 & 98 & 51 & MNA-SF \\
\hline [31] & 594 & 316 & 236 & 42 & MNA-SF \\
\hline [32] & 415 & 152 & 185 & 78 & MNA-SF \\
\hline $\begin{array}{l}\text { Total of } \\
\text { subjects }\end{array}$ & 2195 & 992 & 774 & 411 & \\
\hline Percentage & & $45.2 \%$ & $35.3 \%$ & $18.7 \%$ & \\
\hline
\end{tabular}

$\$$ This screening tool is based on changes in dietary intake, weight, and other risk factors (pressure ulcers, presence of infection, period of fasting, and the need for help with eating and drinking); ${ }^{\dagger}$ Risk of malnutrition cut-off point: Body Mass Index (BMI) between 20 and $22 \mathrm{~kg} / \mathrm{m}^{2}$; $¥$ Mini-Nutritional Assessment (MNA) cut-off points: well-nourished $\geq 24$ points, at risk for malnutrition at 17-23.5 points, and malnourished at less than 17 points; ${ }^{¥}$ Mini-Nutritional Assessment-Short Form (MNA-SF) cut-off points: well-nourished 12-14 points, at risk of malnutrition 8-11 points, and malnourished 0-7 points; $\mathrm{WN}$ : well-nourished; RMN: risk of malnutrition; $\mathrm{MN}$ : malnourished. 
Table 2. Nutritional status and biomarkers in patients with hip fracture.

\begin{tabular}{|c|c|c|c|c|c|}
\hline $\begin{array}{l}\text { Authors } \\
\text { Origin } \\
\text { Publication } \\
\text { Year }\end{array}$ & $\begin{array}{l}\text { Design } \\
\text { Aim } \\
\text { Setting }\end{array}$ & $\begin{array}{l}n \text { (Male/Female) } \\
\text { Age, Mean } \pm \text { SD } \\
\text { (Years) } \\
\text { BMI }\left(\mathrm{kg} / \mathrm{m}^{2}\right)\end{array}$ & $\begin{array}{l}\text { Anthropometry } \\
\text { Measurement of Body } \\
\text { Composition } \\
\text { Biomarkers }\end{array}$ & $\begin{array}{l}\text { (1) Exclusion Criteria } \\
\text { (2) Definition of } \\
\text { Malnutrition }\end{array}$ & Main Outcomes \\
\hline \multirow{2}{*}{$\begin{array}{l}\text { Mansell } \\
\text { UK } \\
1990[33]\end{array}$} & \multirow{2}{*}{$\begin{array}{l}\text { Observational } \\
\text { Comparison of } \\
\text { anthropometric } \\
\text { measurements of women } \\
\text { with HF, with healthy } \\
\text { volunteers in the } \\
\text { community (C) and } \\
\text { patients admitted to } \\
\text { geriatric wards (G) }\end{array}$} & $\begin{array}{l}n 663(0 / 663) \\
\text { HF } 470 \\
\text { Community } 103 \\
\text { Geriatric } 90\end{array}$ & $\begin{array}{l}\text { MAC }(\mathrm{cm}) \\
\text { HF } 22.8 \pm 0.2 \\
\text { Community } 28.6 \pm 0.27 \\
\text { Geriatric } 25.9 \pm 0.41\end{array}$ & \multirow{2}{*}{$\begin{array}{l}\text { (1) For healthy female: } \\
\text { housebound or wheelchairs } \\
\text { (2) NA }\end{array}$} & \multirow{2}{*}{$\begin{array}{l}\text { Fractured group were older than healthy subjects }(p<0.001) . \\
\text { HF vs. Community: } \downarrow \text { MAC } \downarrow \text { AMA } \downarrow \downarrow \text { TSF } \downarrow \downarrow \text { AFA }(p<0.001) \\
\text { Significant MAC reduction per year of age: } \\
-0.20 \pm 0.03 \mathrm{~cm} / \text { year (HF) } \\
-0.15 \pm 0.06 \mathrm{~cm} / \text { year (Community) } \\
\text { Significant TSF reduction per year of age: } \\
-0.16 \pm 0.03 \mathrm{~mm} / \text { year (HF) }\end{array}$} \\
\hline & & $\begin{array}{l}\mathrm{HF}=77.3 \pm 0.3 \text { years } \\
\text { Community } \\
72.5 \pm 0.5 \text { years } \\
\text { Geriatric } \\
79.1 \pm 0.8 \text { years }\end{array}$ & $\begin{array}{l}\text { TSF }(\mathrm{mm}) \\
\text { HF } 13.0 \pm 0.6 \\
\text { Community } 24.7 \pm 0.6\end{array}$ & & \\
\hline $\begin{array}{l}\text { Maffulli } \\
\text { UK } \\
1999[20]\end{array}$ & $\begin{array}{l}\text { Observational } \\
\text { Nutritional differences in } \\
\text { patients with } \\
\text { intertrochanteric (IT) and } \\
\text { intracapsular (IC) } \\
\text { fractures }\end{array}$ & $\begin{array}{l}n 119(91 / 28) \\
\text { IT } 17-54 \\
\text { IC } 11-37 \\
80.8 \pm 9.1 \text { years } \\
21.5 \pm 4.1 \mathrm{~kg} / \mathrm{m}^{2}\end{array}$ & $\begin{array}{l}\text { Intertrochanteric TSF } \\
11.6 \pm 4.5 \mathrm{~mm} \\
\text { BSF } 6.1 \pm 4 \mathrm{~mm} \\
\text { MAC } 23.5 \pm 3.6 \mathrm{~cm} \\
\text { Intracapsular TSF } 10.6 \pm \\
4 \mathrm{~mm} \\
\text { BSF } 5.4 \pm 2.4 \mathrm{~mm} \\
\text { MAC } 21.9 \pm 3.1 \mathrm{~cm}\end{array}$ & $\begin{array}{l}\text { (1) Pathologic fracture } \\
\text { (2) } \mathrm{BMI}<18 \mathrm{~kg} / \mathrm{m}^{2}\end{array}$ & $\begin{array}{l}\text { Malnourished } \rightarrow 45 \% \text { IC vs. } 20 \% \text { IT ( } \mathrm{p}<0.001) \\
19 \% \text { Overweight or obese } \rightarrow 22 \% \text { IT vs. } 2 \% \text { IC } \\
\text { Complications } 15 \% \text { IC vs. } 3 \% \text { IT }(p<0.05) \\
\text { BMI: IC }<\text { IT }\left(20.1 \pm 3.3 \text { vs. } 22.5 \pm 4.6 \mathrm{~kg} / \mathrm{m}^{2}, p<0.01\right)\end{array}$ \\
\hline $\begin{array}{l}\text { Murphy } \\
\text { UK } \\
2000[15]\end{array}$ & $\begin{array}{l}\text { Observational } \\
\text { Assess the sensitivity and } \\
\text { specificity of MNA, } \\
\text { and its comparability with } \\
\text { other nutritional tools }\end{array}$ & $\begin{array}{l}n 49(0 / 49) \\
79.5 \pm 9 \text { years } \\
23.7 \pm 4.3 \mathrm{~kg} / \mathrm{m}^{2}\end{array}$ & Albumin $36.9 \pm 4.7 \mathrm{~g} / \mathrm{L}$ & $\begin{array}{l}\text { (1) Cognitive impairment } \\
\text { (2) MNA }\end{array}$ & $\begin{array}{l}\text { Patients had low mean values for body weight, albumin and } \\
\text { transferrin } \\
\text { Mean energy intake was below the estimated average } \\
\text { requirementMNA < 17: } \\
\text { Sensitivity: } 27-57 \% \\
\text { Specificity: } 66-100 \%\end{array}$ \\
\hline $\begin{array}{l}\text { Lumbers } \\
\text { UK } \\
2001[12]\end{array}$ & $\begin{array}{l}\text { Cross-sectional } \\
\text { Intake and nutritional } \\
\text { status in } \\
\text { HF compared to day } \\
\text { center attendees (DC) }\end{array}$ & $\begin{array}{l}n 125 \\
\text { HF } 75(0 / 75) \\
\text { DC } 50(0 / 50) \\
80.2 \pm 7.9 \text { years } \\
25.5 \pm 4.8 \mathrm{~kg} / \mathrm{m}^{2}\end{array}$ & $\begin{array}{l}\text { HF } \\
\text { MAC } 27.1 \pm 4.3 \mathrm{~cm} \\
\text { TSF } 17 \pm 2.7 \mathrm{~mm} \\
\text { MUAMC } 21.4 \pm 3.4 \mathrm{~cm} \\
\text { Day Centers } \\
\text { MAC } 31.3 \pm 4.7 \mathrm{~cm} \\
\text { TSF } 18.9 \pm 2.8 \mathrm{~mm} \\
\text { MUAMC } 23.3 \pm 3.8 \mathrm{~cm}\end{array}$ & $\begin{array}{l}\text { (1) Mental function test }<7 \\
\text { (2) NA }\end{array}$ & $\begin{array}{l}\text { HF patients vs. day center attendees have: } \\
\text { lower BMI }\left(24.1 \pm 4.7 \mathrm{vs} .27 .5 \pm 4.9 \mathrm{~kg} / \mathrm{m}^{2}, p<0.001\right) \text {; lower } \\
\text { MUAMC, albumin, proteins and energy intake and higher } \mathrm{CRP}(p< \\
0.01) \\
\text { Albumin } \leftrightarrow \operatorname{RCP}(r=-0.45)\end{array}$ \\
\hline $\begin{array}{l}\text { Nematy } \\
\text { UK } \\
2006[14]\end{array}$ & $\begin{array}{l}\text { Observational } \\
\text { Nutritional status and } \\
\text { energy intake }\end{array}$ & $\begin{array}{l}n 25(7 / 18) \\
85.3 \pm 1.5 \text { years } \\
21.9 \pm 1.0 \mathrm{~kg} / \mathrm{m}^{2}\end{array}$ & Albumin $36 \pm 2.6 \mathrm{~g} / \mathrm{L}$ & $\begin{array}{l}\text { (1) Pathological fracture or } \\
\text { elective surgery } \\
\text { (2) Changes in dietary } \\
\text { intake, weight loss, pressure } \\
\text { sore, infection, and need } \\
\text { help for eating }\end{array}$ & $\begin{array}{l}\text { At risk of malnutrition group }(n 17) \text { had lower BMI and lower energy } \\
\text { intake versus well-nourished group }(n 8) \\
\text { BMI: ARM } 19.6 \pm 1.1 \text { vs. WN } 25 \pm 1.5 \mathrm{~kg} / \mathrm{m}^{2} \\
\text { Energy intake: ARM } 3602 \pm 320 \mathrm{vs} \text {. WN } 5044 \pm 528 \mathrm{~kJ} / \text { day }\end{array}$ \\
\hline
\end{tabular}


Table 2. Cont.

\begin{tabular}{|c|c|c|c|c|c|}
\hline $\begin{array}{l}\text { Authors } \\
\text { Origin } \\
\text { Publication } \\
\text { Year }\end{array}$ & $\begin{array}{l}\text { Design } \\
\text { Aim } \\
\text { Setting }\end{array}$ & $\begin{array}{l}n \text { (Male/Female) } \\
\text { Age, Mean } \pm \text { SD } \\
\text { (Years) } \\
\text { BMI }\left(\mathrm{kg} / \mathrm{m}^{2}\right)\end{array}$ & $\begin{array}{l}\text { Anthropometry } \\
\text { Measurement of Body } \\
\text { Composition } \\
\text { Biomarkers }\end{array}$ & $\begin{array}{l}\text { (1) Exclusion Criteria } \\
\text { (2) Definition of } \\
\text { Malnutrition }\end{array}$ & Main Outcomes \\
\hline $\begin{array}{l}\text { Perez } \\
\text { Spain } \\
2010[19]\end{array}$ & $\begin{array}{l}\text { Observational } \\
\text { Prevalence of } \\
\text { malnutrition }\end{array}$ & $\begin{array}{l}n 80(24 / 56) \\
80.6 \pm 6.3 \text { years } \\
27.1 \pm 4.4 \mathrm{~kg} / \mathrm{m}^{2}\end{array}$ & $\begin{array}{l}\text { TSF } 5.5 \pm 2.3 \mathrm{~mm} \\
\text { BSF } 8.1 \pm 4.8 \mathrm{~mm} \\
\text { MAC } 26.8 \pm 3.9 \mathrm{~mm} \\
\text { CC } 31.9 \pm 4 \mathrm{~cm}\end{array}$ & $\begin{array}{l}\text { (1) NA } \\
\text { (2) MNA }\end{array}$ & $\begin{array}{l}\text { Length of hospital stay: men } 15.3 \pm 5.8 \text { days; women } 14.9 \pm 12 \text { days } \\
\text { MNA } \leftrightarrow \text { BMI } r=0.6\end{array}$ \\
\hline \multirow{3}{*}{$\begin{array}{l}\text { Perez } \\
\text { Spain } \\
2011[13]\end{array}$} & \multirow{3}{*}{$\begin{array}{l}\text { Observational } \\
\text { Nutritional status and } \\
\text { intake of HF vs. } \\
\text { community dwelling } \\
\text { study participants }\end{array}$} & $\begin{array}{l}n 86(0 / 86) \\
\mathrm{HF}=44 \\
\text { Community }=42\end{array}$ & \multirow{3}{*}{$\begin{array}{l}\text { MAC (cm) } \\
\text { HF } 27.3 \pm 3.2 \\
\text { Community } 29.1 \pm 4.1 \\
\text { Calf circumference (cm) } \\
\text { HF } 32.5 \pm 3.6 \\
\text { Community } 35.1 \pm 4.4\end{array}$} & \multirow{3}{*}{$\begin{array}{l}\text { (1) No osteoporotic } \\
\text { fractures or major trauma } \\
\text { (2) NA }\end{array}$} & \multirow{3}{*}{$\begin{array}{l}\text { HF has lower BMI, arm and leg circumference than community } \\
\text { dwelling ( } p<0.05) \\
\text { Energy intake (kcal): HF 1417; community dwelling } 2052(p<0.001) \\
\text { Calcium (mg/dL): HF 827; community dwelling } 1265(p<0.001) \\
\text { Vitamin D ( } \mathrm{gg} / \mathrm{dL}): \text { HF 1.6; community dwelling: } 5.2(p<0.001)\end{array}$} \\
\hline & & $\begin{array}{l}\text { Age } \\
\mathrm{HF}=77.9 \pm 4.7 \text { years } \\
\text { Community }= \\
76.2 \pm 4.6 \text { years }\end{array}$ & & & \\
\hline & & 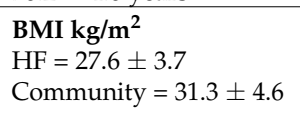 & & & \\
\hline $\begin{array}{l}\text { Koren-Hakim } \\
\text { Israel } \\
2012[18]\end{array}$ & $\begin{array}{l}\text { Retrospective } \\
\text { Association of MNA-SF } \\
\text { with functional status, } \\
\text { comorbidity, } \\
\text { and mortality ( } 36 \text { months) }\end{array}$ & $\begin{array}{l}n 215(61 / 154) \\
83.5 \pm 6.1 \text { years } \\
26.4 \pm 4.9 \mathrm{~kg} / \mathrm{m}^{2}\end{array}$ & $\begin{array}{l}\text { WN28.1 } \pm 4.0 \mathrm{~kg} / \mathrm{m}^{2} \\
\text { ARM } 25.5 \pm 5.1 \mathrm{~kg} / \mathrm{m}^{2} \\
\text { MN } 22.7 \pm 3.7 \mathrm{~kg} / \mathrm{m}^{2}\end{array}$ & $\begin{array}{l}\text { (1) Terminal illnesses and } \\
\text { multi-trauma } \\
\text { (2) MNA }\end{array}$ & $\begin{array}{l}\text { MNA } \leftrightarrow \text { BMI, ADL, cognitive status, readmission, mortality } 36 \mathrm{~m} \text {, } \\
\text { CCI and CIRS-G } \\
\text { Independent variables for mortality } \rightarrow \text { Charlson comorbidity index } \\
\text { and functional status (ADL) }\end{array}$ \\
\hline $\begin{array}{l}\text { Villani } \\
\text { Germany } \\
2013[34]\end{array}$ & $\begin{array}{l}\text { Cross-sectional } \\
\text { Evaluate new screening } \\
\text { tool for detection cachexia }\end{array}$ & $\begin{array}{l}n 71(19 / 52) \\
82.2 \pm 5.8 \text { years } \\
\text { Men } 23.9 \pm 2.9 \mathrm{~kg} / \mathrm{m}^{2} \\
\text { Women } 25.9 \pm 3.8 \\
\mathrm{~kg} / \mathrm{m}^{2}\end{array}$ & $\begin{array}{l}\text { M: } \\
\text { MAC (cm) } 26.7 \pm 3.3 \\
\text { TSF }(\mathrm{mm}) 11.5 \pm 4.8 \\
\text { W: } \\
\text { MAC }(\mathrm{cm}) 27.1 \pm 3.9 \\
\text { TSF }(\mathrm{mm}) 16.4 \pm 5.4\end{array}$ & $\begin{array}{l}\text { (1) Pathological fracture or } \\
\text { malignancy, residing in } \\
\text { residential care } \\
\text { (2) NA }\end{array}$ & $\begin{array}{l}\text { Patients with cachexia: } \\
5 \text { new tool } \\
4 \text { (consensus definition) } \\
\text { New tool: } \\
\text { Sensitivity } 75 \% \text { and specificity } 97 \% \\
\text { Positive predictive value } 60 \% \text {, negative predictive value } 99 \%\end{array}$ \\
\hline $\begin{array}{l}\text { Bell } \\
\text { Australia } \\
2014[35]\end{array}$ & $\begin{array}{l}\text { Prospective } \\
\text { Concurrent and predictive } \\
\text { validity of malnutrition } \\
\text { diagnostic measures }\end{array}$ & $\begin{array}{l}n 142(45 / 97) \\
83.5 \text { years }\end{array}$ & NA & $\begin{array}{l}\text { (1) NA } \\
\text { (2) MNA-SF }<8 \\
\text { BMI }<18.5 \mathrm{~kg} / \mathrm{m}^{2} \\
\mathrm{ALB}<35 \mathrm{~g} / \mathrm{L} \\
\text { ICD10-AM } \\
\text { Geriatrician (subjective } \\
\text { clinical assessment) }\end{array}$ & $\begin{array}{l}\text { Malnutrition prevalence with different tools: BMI }(12.7 \%) \text {, MNA-SF } \\
(27 \%) \text {, ICD10-AM }(48.2 \%), \text { Albumin }(53.2 \%) \text {, subjective assessment } \\
(55.1 \%) \\
\text { MNA-SF } \leftrightarrow \text { ICD10-AM }(r=0.3) \text { and BMI }(r=0.2) \\
\text { ICD10-AM } \leftrightarrow \text { subjective assessment }(r=0.6) \\
\text { ICD10-AM independent predictor of } 4 \text {-month mortality (OR 3.6, } \\
95 \% \text { CI 1.1-11.8) }\end{array}$ \\
\hline
\end{tabular}




\subsection{Influence upon Outcomes and Complications}

The general characteristics of the studies included in this section can be found in Table 3.

Espaulella et al. showed how after 6 months' follow-up only slightly over half of the patients subject to follow-up had recovered the functional status they had before the fracture [36]. The MNA was an independent predictor of functional status upon discharge [30], at four and at 12 months [31]. Malnourished patients are more likely to suffer postoperative delirium [32], as well as other post-operative complications such as sepsis [21] and pressure ulcers [37].

Malnutrition is of double importance as it is a risk factor for hip fracture, and in patients with hip fracture it reduces the ability to recover pre-fracture functional capacity. Indeed, malnutrition is a risk factor for fracture, and malnourished older people generally present a worse functional status before the fracture and frequently recover only partially their pre-fracture level of independency in activities of daily living (ADL) following a hip fracture [27]. Conversely, well-nourished older people tend to improve their functional status at discharge after a hip fracture, as revealed by the motor-Functional Independence Measure (FIM) scale [30].

Malnutrition and risk of malnutrition are more prevalent in geriatric patients with a higher comorbidity [38], in addition to being risk factors for complications following hip fracture surgery, such as pressure ulcers [39].

Albumin could be a good blood marker of malnutrition [40]. In this context, Bohl et al. studied a large database $(17,651$ patients with hip fracture, mean age $84.4 \pm 7.2$ years) and observed a prevalence of malnutrition of $45.9 \%$, defined as albumin values below $3.5 \mathrm{~g} / \mathrm{dL}$ prior to surgery [21]. These authors reported that patients with hypoalbuminemia presented a higher prevalence of sepsis $(p<0.001)$, longer hospital stay $(p<0.001)$, and higher prevalence of readmission $(p=0.054)$. The benefits of a good nutritional status were also observed in other studies [18]. 
Table 3. Association of nutritional status, as revealed by nutritional biomarkers, with outcomes and post-operative complications.

\begin{tabular}{|c|c|c|c|c|c|}
\hline $\begin{array}{l}\text { Authors } \\
\text { Origin }\end{array}$ & $\begin{array}{l}\text { Design } \\
\text { Aim }\end{array}$ & $\begin{array}{l}n \text { (Male/Female) } \\
\text { Age, Mean } \pm \text { SD (Years) }\end{array}$ & $\begin{array}{l}\text { BMI }\left(\mathrm{kg} / \mathrm{m}^{2}\right) \\
\text { Biomarkers }\end{array}$ & $\begin{array}{l}\text { Exclusion Criteria } \\
\text { MN Definition Tool }\end{array}$ & Main Outcomes \\
\hline $\begin{array}{l}\text { Formiga } \\
\text { Spain } \\
2005[41]\end{array}$ & $\begin{array}{l}\text { Prospective } \\
\text { observational } \\
\text { Relationship } \\
\text { between } \\
\text { nutritional status } \\
\text { and complications }\end{array}$ & $\begin{array}{l}n 73(12 / 61) \\
81.5 \pm 7.1 \text { years }\end{array}$ & $\begin{array}{l}\text { Cholesterol } 4.3 \pm 1.1 \mathrm{mmol} / \mathrm{L} \\
\text { Albumin } 30.6 \pm 3.6 \mathrm{~g} / \mathrm{L} \\
\mathrm{TLC} / \mathrm{mm}^{3} 1278 \pm 463\end{array}$ & $\begin{array}{l}\text { Pathological or multiple } \\
\text { fractures, terminally ill } \\
\text { patients, surgery delayed } \\
>48 \mathrm{~h} \text { or lipid-lowering drug } \\
\text { MNA-SF }<11\end{array}$ & $\begin{array}{l}\text { MNA-SF } \rightarrow 11 \pm 0.5 \\
\text { MNA-SF not predict } \rightarrow \text { nosocomial infections and } \\
\text { pressure ulcers } \\
\text { Albumin predict } \rightarrow \text { nosocomial infections } \\
\downarrow \text { TLC years } \downarrow \text { Albumin predict } \rightarrow \text { pressure ulcers } \\
\text { Barthel index } \leftrightarrow \text { Charlson comorbidity index } r=-0.9 \\
(p<0.0001) \\
\text { Length of hospital stay }=16.4 \text { days }\end{array}$ \\
\hline $\begin{array}{l}\text { Montero } \\
\text { Spain } \\
2007[42]\end{array}$ & $\begin{array}{l}\text { Prospective cohort } \\
\text { Relationship } \\
\text { between } \\
\text { malnutrition and } \\
\text { recovery }\end{array}$ & $\begin{array}{l}n 110(22 / 88) \\
81.4 \pm 7.3 \text { years }\end{array}$ & $\begin{array}{l}25(\mathrm{OH}) \mathrm{vitD} 10.8 \pm 5.3 \mathrm{ng} / \mathrm{ml} \\
\mathrm{TLC} / \mathrm{mm}^{3} 1545 \pm 592 \\
\text { Albumin } 32.6 \pm 3.8 \mathrm{~g} / \mathrm{L} \\
\text { Prealbumin } 15.3 \pm 4.7 \mathrm{mg} / \mathrm{dL} \\
\text { Cholesterol } \\
160.5 \pm 40.8 \mathrm{mg} / \mathrm{dL} \\
\text { Transferrin } \\
195.9 \pm 47.1 \mathrm{mg} / \mathrm{dL}\end{array}$ & $\begin{array}{l}\text { Pathologic or major trauma } \\
\text { fractures } \\
\text { Anthropometric and blood } \\
\text { biomarkers }\end{array}$ & $\begin{array}{l}38.8 \% \text { regained pre-fracture functional state } \\
\text { Dementia } \leftrightarrow \downarrow \text { functional recovery } \\
25(\mathrm{OH}) \mathrm{vit} \mathrm{D}<10 \mathrm{ng} / \mathrm{ml} \leftrightarrow \downarrow \text { pre-fracture functional } \\
\text { state, with bedridden }(1 \text { year) and with no functional } \\
\text { recovery }(p<0.05) \\
\text { Factors associated to bedridden ( } 1 \text { year) } \mathrm{OR}, 95 \% \mathrm{CI} \\
\text { - } \quad \text { pre-fracture functional status } 10.02,2.83-35.47 \\
\quad p<0.01 \\
\quad \text { Caloric malnutrition } 9.57(2.18-42.84) p<0.01 \\
\text { - } \quad \text { Protein malnutrition } 15.23(1.36-1.70) p<0.05\end{array}$ \\
\hline $\begin{array}{l}\text { Baumgarten } \\
\text { USA } \\
2009 \text { [37] }\end{array}$ & $\begin{array}{l}\text { Prospective cohort } \\
\text { Identify care } \\
\text { settings associated } \\
\text { with increased } \\
\text { pressure ulcers } \\
\text { risk }\end{array}$ & $\begin{array}{l}n 658(152 / 506) \\
83.2 \pm 6.6 \text { years }\end{array}$ & $23.8 \pm 5.1 \mathrm{~kg} / \mathrm{m}^{2}$ & $\begin{array}{l}\text { Fractures occurred during } \\
\text { hospital stay } \\
\text { Subjective Global Assessment } \\
\text { (SGA) }\end{array}$ & $\begin{array}{l}\text { Pressure ulcers at baseline } \leftrightarrow \uparrow \text { severe illness, } \uparrow \\
\text { comorbidity, } \downarrow \text { nutritional status, } \downarrow \text { cognitive status } \\
(p<0.05) \\
\text { Albumin }<30 \mathrm{~g} / \mathrm{L}: 31.5 \% \\
\text { Length of hospital stay } 5.6 \pm 2.8 \text { (no pressure ulcers) } \\
\text { vs. } 6.6 \pm 3.8 \text { (pressure ulcers) }(p<0.001)\end{array}$ \\
\hline $\begin{array}{l}\text { Drevet } \\
\text { France } \\
2014[26]\end{array}$ & $\begin{array}{l}\text { Prospective } \\
\text { observational } \\
\text { Protein Energy } \\
\text { Malnutrition } \\
\text { prevalence }\end{array}$ & $\begin{array}{l}n 50(15 / 35) \\
86.1 \pm 4.4 \text { years }\end{array}$ & $22.6 \pm 4.3 \mathrm{~kg} / \mathrm{m}^{2}$ & $\begin{array}{l}\text { Road accident } \\
\text { MNA }\end{array}$ & $\begin{array}{l}\text { Prevalence of PEM was } 28 \%(n 14) \\
\text { Mean hospital stay: PEM } 21.9 \pm 16.7 \text { vs. } 13.4 \pm 6.7 \text { in } \\
\text { non-PEM }(p=0.012)\end{array}$ \\
\hline $\begin{array}{l}\text { Goisser } \\
\text { Germany } \\
2015[27]\end{array}$ & $\begin{array}{l}\text { Observational } \\
\text { Relationship } \\
\text { between } \\
\text { nutritional status } \\
\text { (MNA) and } \\
\text { functional and } \\
\text { clinical course }\end{array}$ & $\begin{array}{l}n 97(20 / 77) \\
84 \pm 5 \text { years }\end{array}$ & NA & $\begin{array}{l}\text { Terminal state, cancer-related } \\
\text { pathologic fractures, cancer } \\
\text { with acute radiation or } \\
\text { chemotherapy } \\
\text { MNA }\end{array}$ & $\begin{array}{l}\text { Patients at risk for malnutrition and malnourished: } \\
-\quad \text { Baseline, } \uparrow \text { comorbidities } \uparrow \text { Charlson } \\
\text { comorbidity index } \uparrow \text { pressure ulcers } \downarrow \text { cognitive } \\
\text { status }(p<0.05) \\
-\quad \text { All times, } \downarrow \text { ADL score }(p<0.05) \\
-\quad 68 \% \text { did not regain pre-fracture ADL } \\
-\quad 18 \% \text { did not regain pre-fracture mobility level } \\
(p=0.02)\end{array}$ \\
\hline
\end{tabular}


Table 3. Cont.

\begin{tabular}{|c|c|c|c|c|c|}
\hline $\begin{array}{l}\text { Authors } \\
\text { Origin }\end{array}$ & $\begin{array}{l}\text { Design } \\
\text { Aim }\end{array}$ & $\begin{array}{l}n \text { (Male/Female) } \\
\text { Age, Mean } \pm \text { SD (Years) }\end{array}$ & $\begin{array}{l}\text { BMI }\left(\mathrm{kg} / \mathrm{m}^{2}\right) \\
\text { Biomarkers }\end{array}$ & $\begin{array}{l}\text { Exclusion Criteria } \\
\text { MN Definition Tool }\end{array}$ & Main Outcomes \\
\hline $\begin{array}{l}\text { Bohl } \\
\text { USA } \\
2017 \text { [21] }\end{array}$ & $\begin{array}{l}\text { Retrospective } \\
\text { Association } \\
\text { between albumin } \\
\text { with death, } \\
\text { and postoperative } \\
\text { complications }\end{array}$ & $\begin{array}{l}n 17,651(12,595 / 5056) \\
84.4 \pm 7.2 \text { years }\end{array}$ & $\begin{array}{l}24.6 \pm 5.6 \mathrm{~kg} / \mathrm{m}^{2} \\
\text { Albumin } 35 \pm 5 \mathrm{~g} / \mathrm{dL}\end{array}$ & $\begin{array}{l}\text { Preoperative serum albumin } \\
\text { concentration not available } \\
\text { Albumin concentration }\end{array}$ & $\begin{array}{l}18.5 \% \text { had BMI }<20 \mathrm{~kg} / \mathrm{m}^{2} \\
\text { Patients with hypoalbuminemia had higher rates: } \\
-\quad \begin{array}{l}\text { of death (RR } 1.52 .95 \% \text { CI } 1.37-1.70 . p<0.001) \\
\text { - }\end{array} \quad \begin{array}{l}\text { of sepsis (RR } 1.92 .95 \% \text { CI } 1.36-2.72 . p<0.001) \\
\text { of longer legnth of hospital stay, } 5.7 \pm 4.7 \mathrm{vs.}\end{array} \\
5.0 \pm 3.9 \text { days }(p<0.001)\end{array}$ \\
\hline $\begin{array}{l}\text { Helminen } \\
\text { Finland } \\
2017 \text { [31] }\end{array}$ & $\begin{array}{l}\text { Prospective } \\
\text { Prognostic } \\
\text { significance of } \\
\text { MNA and albumin }\end{array}$ & $\begin{array}{l}n 594(169 / 425) \\
84 \text { years }\end{array}$ & $\begin{array}{l}24.9 \mathrm{~kg} / \mathrm{m}^{2} \\
\text { Albumin } 33.5 \mathrm{~g} / \mathrm{L}\end{array}$ & $\begin{array}{l}\text { Pathological or periprosthetic } \\
\text { fractures, institutionalization, } \\
\text { prefecture inability to walk } \\
\text { MNA-SF }\end{array}$ & $\begin{array}{l}\text { All nutritional measures were significantly associated } \\
\text { with mortality } \\
\text { Being at risk for malnutrition or being malnourished } \\
\text { were significantly associated with impaired mobility } \\
\text { at } 4 \text { months and } 1 \text { year }\end{array}$ \\
\hline $\begin{array}{l}\text { Mazzola } \\
\text { Italy } \\
2017 \text { [32] }\end{array}$ & $\begin{array}{l}\text { Prospective } \\
\text { If nutritional } \\
\text { status predict } \\
\text { postoperative } \\
\text { delirium }\end{array}$ & $\begin{array}{l}n 415(104 / 309) \\
84 \pm 6.6 \text { years }\end{array}$ & $\begin{array}{l}\text { NA } \\
\text { Albumin } 33 \pm 5.4 \mathrm{~g} / \mathrm{L}\end{array}$ & $\begin{array}{l}\text { Nonoperative approach and } \\
\text { preoperative delirium } \\
\text { MNA-SF }\end{array}$ & $\begin{array}{l}\text { Risk to develop postoperative delirium: } \\
\text { - } \quad \text { at risk for malnutrition: OR } 2.42,95 \% \mathrm{CI} \\
1.29-4.53 \\
\text { - } \quad \text { malnourished: OR } 2.98,95 \% \mathrm{CI} 1.43-6.19\end{array}$ \\
\hline $\begin{array}{l}\text { Inoue } \\
\text { Japan } \\
2017 \text { [30] }\end{array}$ & $\begin{array}{l}\text { Prospective } \\
\text { Relationship } \\
\text { between } \\
\text { nutritional status } \\
\text { and functional } \\
\text { recovery }\end{array}$ & $\begin{array}{l}204(39 / 165) \\
82.7 \pm 9.2 \text { years }\end{array}$ & $\begin{array}{l}20.2 \pm 2.5 \mathrm{~kg} / \mathrm{m}^{2} \\
\text { Albumin } 36 \pm 9 \mathrm{~g} / \mathrm{L}\end{array}$ & $\begin{array}{l}\text { Terminal disease, chronic } \\
\text { liver disease, pre-fracture } \\
\text { ambulation difficulty, no } \\
\text { weight-bearing, discontinued } \\
\text { postoperative rehabilitation } \\
\text { MNA-SF }\end{array}$ & $\begin{array}{l}\text { Well-nourished had higher motor-FIM score at } \\
\text { discharge } \\
\text { Motor-FIM at discharge was significant associated } \\
\text { with MNA-SF }\end{array}$ \\
\hline
\end{tabular}

ADL: activities of daily living; BMI: body mass index; FIM: functional Independence Measure; HF: hip fracture; MNA: Mini Nutritional Assessment; PEM: protein energy malnutrition; OR: odd-ratio; $95 \%$ CI: $95 \%$ confidence interval. $\leftrightarrow$ : correlation. 


\subsection{Malnutrition and Mortality in Older People with Hip Fractures}

In this section we included those studies whose main objective was to assess the impact of malnutrition, as revealed by nutritional biomarkers, on mortality. In addition, we considered studies where a multivariable analysis was carried out and which included malnutrition biomarkers. A summary of the design, characteristics, and main results of the included studies can be found in Table 4 . We included five studies, with a total of 2518 patients ( $71.8 \%$ females), mean age $84.3 \pm 7.2$ years.

Mortality was inversely associated with pre-surgery albumin levels, and patients with hypoalbuminemia had a relative risk of dying of 1.52 (95\% Confidence Interval (CI) 1.37-1.70, $p<0.001$ ) [21]. Regardless of the tool used to diagnose malnutrition, low values of albumin or BMI or low MNA were associated with an increase in mortality. Albumin concentrations of less than $36 \mathrm{~g} / \mathrm{L}$ were associated with a 4-year mortality nearly six times greater (Odd-Ratio (OR) 5.85, 95\% CI 2.3-16.5) [43]. Furthermore, BMI values of less than $22 \mathrm{~kg} / \mathrm{m}^{2}$ were associated with an increase of almost seven times the mortality at 1 year, as compared to values higher than $25 \mathrm{~kg} / \mathrm{m}^{2}$ (Hazard Ratio (HR) 7.25, 95\% CI 1.6-33.7) [22]. Studies such as that of Flodin and collaborators confirm the anterior outcome, observing that subjects with a BMI greater than $26 \mathrm{~kg} / \mathrm{m}^{2}$ had a risk almost three times less of dying after 1 year from the fracture (OR 2.6, 95\%CI 1.4-5.0) [44].

Cenzer et al. showed that difficulty preparing meals after hip fracture predicts 1-year mortality (and this predictor factor has the same points as congestive heart failure) [45]. Others factors such as age, male sex, congestive heart failure, and not being able to drive complete the risk stratification scale [45].

Mortality increases progressively after a hip fracture, from an in-hospital mortality of $7 \%$, to $11 \%$ in the first 6 months after the fracture, up to $30 \%$ in the first year and $40 \%$ at 3 years. To highlight the importance of this health problem, we summarized total mortality and the follow-up periods of the included studies in Table 5. 
Table 4. Relationship between nutritional status and mortality.

\begin{tabular}{|c|c|c|c|c|}
\hline $\begin{array}{l}\text { Authors } \\
\text { Origin } \\
\text { Year } \\
\text { Design }\end{array}$ & $\begin{array}{l}n(\text { Male/Female) } \\
\text { Age, Mean } \pm \text { SD (Years) }\end{array}$ & $\begin{array}{l}\text { BMI kg/m² } \\
(\text { Mean } \pm \text { SD) }\end{array}$ & Exclusion Criteria & Main Outcomes \\
\hline $\begin{array}{l}\text { Miyanishi } \\
\text { Japan } \\
2010 \\
\text { Retrospective [43] }\end{array}$ & $\begin{array}{l}n 129(24 / 103) \\
79 \text { years } \\
\text { Survivors } 78 \pm 11 \text { years } \\
\text { Non-survivors } 81 \pm 10 \text { years }\end{array}$ & $\begin{array}{l}21 \pm 2.9 \\
\text { (Survivors) } \\
18.9 \pm 3.5 \text { (Non } \\
\text { Survivors) }\end{array}$ & NA & $\begin{array}{l}\text { Non-survivors have: } \\
\downarrow^{*} \text { BMI, hemoglobin, albumin and } \uparrow^{*} \text { dementia, complications } \\
\text { Mortality predictors }(4 \text {-year mortality): } \\
\text { Albumin }(<36 \mathrm{~g} / \mathrm{L}) \mathrm{OR}=5.85 \text { and } \mathrm{BMI}\left(<18.9 \mathrm{~kg} / \mathrm{m}^{2}\right) \text {; OR }=1.16\end{array}$ \\
\hline $\begin{array}{l}\text { Schaller } \\
\text { Switzerland } \\
2012 \\
\text { Sub-analysis of } \\
\text { RCT [22] }\end{array}$ & $\begin{array}{l}n 173(36 / 137) \\
84.2 \pm 6.7 \text { years }\end{array}$ & NA & $\begin{array}{l}\text { Severe cognitive } \\
\text { impairment }(\text { MMSE }>15) \text { or } \\
\text { delirium }\end{array}$ & $\begin{array}{l}\text { Risk factor for } \uparrow \text { mortality (1-year mortality): } \\
\text { MMSE }<25 \text { (HR = 5.77, 95\%CI: } 1.55-21.55) \\
\text { Male sex (HR }=3.55,95 \% \text { CI: } 1.26-97) \\
\text { BMI }<22 \text { vs. }>25 \text { (HR = 7.25, 95\%CI: } 1.61-33.74) \\
\text { Vitamin D per } 1 \mathrm{ng} / \mathrm{ml}(\mathrm{HR}=0.93,95 \% \mathrm{CI}: 0.87-0.998)\end{array}$ \\
\hline $\begin{array}{l}\text { Gumieiro } \\
\text { Brasil } \\
2013 \\
\text { Prospective [46] }\end{array}$ & $\begin{array}{l}n 86(20 / 66) \\
80.2 \pm 7.3 \text { years }\end{array}$ & NA & Pathological fracture & $\begin{array}{l}\text { MNA } \leftrightarrow \text { gait impairment } \mathrm{OR}=0.77(0.66-0.90) p=0.001 \\
\uparrow 1 \text { point } \mathrm{MNA} \rightarrow \uparrow * 29 \% \text { chance of walking } \\
\text { MNA } \leftrightarrow \text { mortality } \mathrm{HR}=0.87(0.76-0.99) p=0.04 \\
\uparrow 1 \text { point MNA } \rightarrow \downarrow^{*} 15 \% \text { mortality risk }\end{array}$ \\
\hline $\begin{array}{l}\text { Flodin } \\
\text { Sweden } \\
2016 \\
\text { Prospective [44] }\end{array}$ & $\begin{array}{l}n 843(227 / 616) \\
82 \pm 7 \text { years }\end{array}$ & $22.7 \pm 3.8 \mathrm{~kg} / \mathrm{m}^{2}$ & $\begin{array}{l}\text { Severe cognitive } \\
\text { impairment, admitted from } \\
\text { nursing-homes }\end{array}$ & $\begin{array}{l}\text { 1-year mortality }(p=0.006) \text { : } \\
\text { BMI }>26=6 \% \\
\text { BMI } 22-26=18 \% \\
\text { BMI }<22=16 \% \\
\text { BMI }>26 \text { indicates a higher likelihood of returning to independen } \\
\text { living (OR } 2.6,95 \% \text { CI } 1.4-5.0)\end{array}$ \\
\hline $\begin{array}{l}\text { Uriz-Otano } \\
\text { Spain } \\
2016 \\
\text { Prospective [47] }\end{array}$ & $\begin{array}{l}n 430(97 / 333) \\
84.2 \pm 7.4 \text { years }\end{array}$ & NA & Tumor, high impact fracture & $\begin{array}{l}\text { 3-year mortality: } \\
\text { Albumin HR 0.61, 95\%CI 0.42-0.90 } \\
\text { Predictors of 3-year mortality: } \\
\text { Age, HR 1.04, 95\%CI 1.01-1.06 } \\
\text { Comorbidity, HR 1.19, 95\%CI 1.09-1.30 } \\
\text { Complications, HR 1.17, 95\%CI 1.05-1.31 }\end{array}$ \\
\hline
\end{tabular}

MMSE: Mini-Mental State Examinatio; RCT: randomized clinical trial; $\downarrow^{*}$ : significantly less; $\uparrow^{*}$ : significantly more. 
Table 5. Total mortality during hospital stay, and at various stages after discharge.

\begin{tabular}{|c|c|c|c|c|c|c|}
\hline Reference & In-Hospital & $<6$ Months & 1 Year & 36 Months & $>36$ Months & $n$ \\
\hline [18] & $6 \%$ & & & $36.7 \%$ & & 215 \\
\hline [20] & $6 \%$ & & & & & 119 \\
\hline [21] & $7.4 \%$ & & & & & 17,651 \\
\hline [22] & & & $27 \%$ & & & 173 \\
\hline [27] & $15 \%$ & & & & & 97 \\
\hline [29] & & $7.70 \%$ & & & & 152 \\
\hline [31] & & $30 \%$ & $26 \%$ & & & 594 \\
\hline [35] & $4.9 \%$ & $14.8 \%$ & & & & 142 \\
\hline [36] & $4 \%$ & $21.1 \%$ & & & & 171 \\
\hline [39] & & $29.1 \%$ & $42.40 \%$ & & & 420 \\
\hline [41] & $10 \%$ & & & & & 73 \\
\hline [42] & $6.4 \%$ & $11.8 \%$ & $19.4 \%$ & & & 110 \\
\hline [43] & & & & & $48 \%$ & 129 \\
\hline [45] & & & $27 \%$ & & & 857 \\
\hline [46] & & $12.8 \%$ & & & & 86 \\
\hline [48] & $1.7 \%$ & $17.9 \%$ & & & & 57 \\
\hline [49] & $11.6 \%$ & $20.6 \%$ & & & & 302 \\
\hline Total mortality (\%) & $7.4 \%$ & $20.4 \%$ & $29.3 \%$ & $39.4 \%$ & $48 \%$ & 23,093 \\
\hline
\end{tabular}

\subsection{Effects of Nutritional Intervention}

In this section we included the studies in which nutritional interventions were carried out. The general characteristics of the populations included, the design, and the main results of the studies included are presented in Table 6.

We included 18 studies, 14 of which were carried out in Europe, one in the USA, one in Australia, and two in Asia, totaling 2248 patients (each study including between 23 and 420 subjects), with an mean age of $81.6 \pm 5.4$. Five studies were carried out only on women, whereas the rest had mixed samples (66.8\% women).

A majority of the studies ( $n$ 14) used oral nutritional supplements. One was preceded by supplementation with parenteral nutrition. In one study the supplement was administered via naso-gastric tube, and in one other study only dietary advice was used. One study did not specify the type of intervention. The characteristics of the interventions, calories used, protein content, and duration of the treatment are summarized in Table 7.

Regarding duration, in seven studies intervention was maintained during hospital stay, in four studies the duration was $\leq 3$ months, and in two it was up to 6 months. Two of the studies did not specify the duration of treatment.

The results demonstrated that a good compliance in the use of oral supplements was associated with an increase in total energy, protein, and liquid intake during hospital stay $[16,17,23,24,48]$. This is important because higher nutritional intake was associated with less postoperative complications. This improvement in intake brought on an increase of IGF-1, a decrease of bone loss 1 year after the fracture [50], lesser prevalence and intensity of delirium, and lower production of oxidative stress-derived products [23]. Nutritional supplementation could also lead to a decrease in the incidence and duration of pressure ulcers, as well as delay their onset. Weight loss was found among subjects who received no supplementation (the control group) [25,51,52], probably due to a loss of muscle mass mboxciteB53-nutrients-273625,B54-nutrients-273625. Two recent studies used supplements enriched with Calcium $\beta$-Hydroxy- $\beta$-Methylbutyrate (CaHMB); these studies observed an improvement in muscle indices in the intervention groups but no improvement in the control groups [54,55].

A multidisciplinary approach is required in order to reduce malnourishment in subjects admitted to hospital [25]. Having a dietician on the team [49] as well as nurses trained in nutrition [25] was associated with an increase in energy, protein, and supplement intake. In addition, a multidisciplinary approach was shown to counteract increases in the incidence of malnutrition after discharge [25]. Furthermore, nutritional intervention was associated with lower short- and long-term mortality rate as well as with an increase in quality of life (as revealed by the EuroQol-5D scale) [25,49]. Nutritional advice for well-nourished patients was associated with better performance in the ADL, and with a better recovery of the ability to walk [28]. 
Table 6. Nutritional intervention in patients with hip fracture.

\begin{tabular}{|c|c|c|c|c|c|}
\hline $\begin{array}{l}\text { Author } \\
\text { Year } \\
\text { Origin }\end{array}$ & $\begin{array}{l}\text { Design } \\
\text { Aim }\end{array}$ & $\begin{array}{l}n \text { (Male/Female) } \\
\text { Age, Mean } \pm \text { SD } \\
\text { (Years) } \\
\text { Follow-Up (FU) }\end{array}$ & $\begin{array}{l}\text { BMI kg/m² } \\
(\text { Mean } \pm \text { SD) } \\
\text { Measurement of Body } \\
\text { Composition }\end{array}$ & Exclusion Criteria & Results \\
\hline $\begin{array}{l}\text { Schürch [50] } \\
1998 \\
\text { Switzerland }\end{array}$ & $\begin{array}{l}\text { RCT } \\
\text { Effects of oral protein } \\
\text { supplements on bone } \\
\text { metabolism }\end{array}$ & $\begin{array}{l}n 82(8 / 74) \\
\text { IG } 41 \\
\text { CG } 41 \\
80.7 \pm 7.4 \text { years } \\
6 \text { months }\end{array}$ & $\begin{array}{l}24.3 \pm 4.0 \mathrm{~kg} / \mathrm{m}^{2} \\
\text { MAC }(\mathrm{cm}) \\
24.1 \pm 3.1\end{array}$ & $\begin{array}{l}\text { Pathologic fracture, fracture caused by severe } \\
\text { trauma, history of contralateral hip fracture, } \\
\text { severe mental impairment, bone disease, renal } \\
\text { failure, and life expectancy }<1 \text { year }\end{array}$ & $\begin{array}{l}\text { IG (at } 6 \mathrm{~m}): \\
\downarrow \text { rehabilitation stay ( } 42.2 \pm 6.6 \text { vs. } \\
53 \pm 4.6 \text { days) } p=0.018 \\
\uparrow \text { increase IGF- } 1 \text { and IgM } p<0.05 \\
50 \% \text { reduction of proximal femur bone loss } \\
\text { (1 year) }\end{array}$ \\
\hline $\begin{array}{l}\text { Espaulella [36] } \\
2000 \\
\text { Spain }\end{array}$ & $\begin{array}{l}\text { RCT } \\
\text { Nutritional supplement and } \\
\text { functional recovery, } \\
\text { complications and mortality }\end{array}$ & $\begin{array}{l}n 171(36 / 135) \\
\text { IG } 85 \\
\text { CG } 86 \\
82.6 \pm 6.6 \text { years } \\
\text { Follow-up: } \\
6 \text { months }\end{array}$ & $\begin{array}{l}25.4 \pm 5 \mathrm{~kg} / \mathrm{m}^{2} \\
\text { MAC: } 24.6 \pm 3.8 \mathrm{~cm} \\
\text { Albumin: } 35 \pm 5.5 \mathrm{~g} / \mathrm{L}\end{array}$ & $\begin{array}{l}\text { Advanced dementia, intravenous nutrition, } \\
\text { pathologic fractures, and accidental falls }\end{array}$ & $\begin{array}{l}\text { Patients with } \geq 1 \text { complication ( } 6 \text { months): } \\
\text { IG } 44(55 \%) \\
\text { CG } 57(70.4 \%) p=0.04 \\
\text { IG: } \uparrow \text { increase albumin ( } 3 \text { months and } \\
6 \text { months) }\end{array}$ \\
\hline $\begin{array}{l}\text { Bruce [51] } \\
2003 \\
\text { Australia }\end{array}$ & $\begin{array}{l}\text { RCT } \\
\text { Nutritional supplements and } \\
\text { prevention of weight loss and } \\
\text { improvement of outcomes }\end{array}$ & $\begin{array}{l}n 109(0 / 109) \\
\text { IG } 50 \\
\text { CG } 59 \\
83.9 \pm 7.7 \text { years } \\
\text { Follow-up: } \\
6 \text { months }\end{array}$ & $\begin{array}{l}22.8 \pm 2.6 \mathrm{~kg} / \mathrm{m}^{2} \\
\text { Albumin } 38.8 \pm 4.1 \mathrm{~g} / \mathrm{L}\end{array}$ & $\begin{array}{l}\mathrm{BMI}<20 \text { or } \mathrm{BMI}>30 \mathrm{~kg} / \mathrm{m}^{2} \text {, residents of } \\
\text { nursing homes, diseases that influence } \\
\text { nutritional intake, diabetes, and fracture due } \\
\text { to a major trauma }\end{array}$ & $\begin{array}{l}\text { Weight loss (all patients): } \\
\text { At } 4 \text { weeks } \\
31.5 \%>5 \% \text { weight loss } \\
20.7 \%>7.5 \% \text { weight loss } \\
\text { At } 8 \text { weeks } \\
27.4 \%>5 \% \text { weight loss } \\
14.6 \%>5 \% \text { weight loss } \\
\text { Fewer weight loss } \leftrightarrow \uparrow \text { number of cane } \\
(p=0.019) \text { and } \uparrow \text { duration of } \\
\text { supplementation }(p<0.05)\end{array}$ \\
\hline $\begin{array}{l}\text { Houwing [56] } \\
2003 \\
\text { The Netherlands }\end{array}$ & $\begin{array}{l}\text { RCT } \\
\text { Effect of a high-protein } \\
\text { supplement on the } \\
\text { development of pressure } \\
\text { ulcers }\end{array}$ & $\begin{array}{l}n 103(19 / 84) \\
81.0 \pm 1.1 \text { years }\end{array}$ & $23.9 \pm 0.5 \mathrm{~kg} / \mathrm{m}^{2}$ & $\begin{array}{l}\text { Terminal care, metastatic hip fracture, } \\
\text { insulin-dependent diabetes, renal disease, } \\
\text { hepatic disease, } \mathrm{BMI}>40 \mathrm{~kg} / \mathrm{m}^{2} \text {. }\end{array}$ & $\begin{array}{l}55.3 \% \text { developed pressure ulcers stage I or II. } \\
\text { Incidence of pressure ulcers stage II: } \\
\text { supplement } 18 \% \text {, placebo } 28 \% \\
57 \% \text { of patients developed pressure ulcers by } \\
\text { the second day }\end{array}$ \\
\hline $\begin{array}{l}\text { Sullivan [48] } \\
2004 \\
\text { USA }\end{array}$ & $\begin{array}{l}\text { RCT } \\
\text { Efficacy of enteral nutrition to } \\
\text { decrease complications and } \\
\text { long-term outcomes }\end{array}$ & $\begin{array}{l}n 57(39 / 19) \\
\text { IG } 27 \\
\text { CG } 30 \\
79 \pm 7.6 \text { years } \\
\text { Follow-up: } \\
6 \text { months }\end{array}$ & $\begin{array}{l}22.1 \pm 4.4 \mathrm{~kg} / \mathrm{m}^{2} \\
\text { BSF: } 6.4 \pm 3.3 \mathrm{~mm} \\
\text { Albumin: } 33.9 \pm 4.5 \mathrm{~g} / \mathrm{L}\end{array}$ & $\begin{array}{l}\text { Pathological fracture, significant trauma to } \\
\text { other organ systems, metastatic cancer, } \\
\text { cirrhosis of the liver, and organ failure }\end{array}$ & $\begin{array}{l}\text { IG: } \\
\uparrow \text { intake of total nutrients } p=0.012 \\
\text { At discharge: } \\
\uparrow \text { Albumin: IG } 29 \pm 5 \text { vs. CG } 25 \pm 5 \mathrm{~g} / \mathrm{L} \\
p=0.002\end{array}$ \\
\hline
\end{tabular}


Table 6. Cont

\begin{tabular}{|c|c|c|c|c|c|}
\hline $\begin{array}{l}\text { Author } \\
\text { Year } \\
\text { Origin }\end{array}$ & $\begin{array}{l}\text { Design } \\
\text { Aim }\end{array}$ & $\begin{array}{l}n \text { (Male/Female) } \\
\text { Age, Mean } \pm \text { SD } \\
\text { (Years) } \\
\text { Follow-Up (FU) }\end{array}$ & $\begin{array}{l}\mathrm{BMI} \mathrm{kg} / \mathrm{m}^{2} \\
(\text { Mean } \pm \text { SD) } \\
\text { Measurement of Body } \\
\text { Composition }\end{array}$ & Exclusion Criteria & Results \\
\hline $\begin{array}{l}\text { Tidermark [53] } \\
\text { 2004 } \\
\text { Sweden }\end{array}$ & $\begin{array}{l}\text { RCT } \\
\text { Effects of nutritional } \\
\text { treatment on nutritional and } \\
\text { functional status }\end{array}$ & $\begin{array}{l}n 60(0 / 60) \\
82.9 \pm 5.4 \text { years } \\
\text { Follow-up: } \\
12 \text { months }\end{array}$ & $20.4 \pm 2.3 \mathrm{~kg} / \mathrm{m}^{2}$ & $\begin{array}{l}<70 \text { years, BMI }>24 \mathrm{~kg} / \mathrm{m}^{2} \text {, cognitive } \\
\text { impairment and institutionalized, dependent } \\
\text { to walk, fractures older than } 24 \mathrm{~h} \text {, pathological } \\
\text { fractures, rheumatoid arthritis. }\end{array}$ & $\begin{array}{l}\text { Lean body mass decreased in the CG and } \\
\text { protein groups, but remained the same in the } \\
\text { protein plus nandrolone group. } \\
\text { ADL declined only in the CG. }\end{array}$ \\
\hline $\begin{array}{l}\text { Eneroth [16] } \\
2005 \\
\text { Sweden }\end{array}$ & $\begin{array}{l}\text { RCT } \\
\text { Effects of nutritional } \\
\text { supplements on nutritional } \\
\text { status and intake. }\end{array}$ & $\begin{array}{l}n 80 \\
\text { IG } 40(7 / 33) \\
\text { CG } 40(10 / 30) \\
81.4 \pm 7.6 \text { years }\end{array}$ & $23.9 \pm 3.8 \mathrm{~kg} / \mathrm{m}^{2}$ & $\begin{array}{l}\text { Multiple and pathologic fractures, malignant } \\
\text { disease, inflammatory joint disease, dementia, } \\
\text { depression, acute psychosis, epileptic seizures, } \\
\text { insulin-treated diabetes mellitus, heart, kidney, } \\
\text { or liver insufficiency }\end{array}$ & $\begin{array}{l}\text { PEM baseline: } \\
\text { CG 33\%, IG } 38 \% \\
\text { Fluid intake: } \\
\text { IG }=1856 \mathrm{ml}, \mathrm{CG}=1300 \mathrm{ml}(p<0.0001) \\
\text { Energy intake during days } 1-10: \\
\text { IG = } 1296 \mathrm{kcal} / \text { day CG }=916 \mathrm{kcal} / \text { day } \\
(p=0.003) \\
\text { Difference between actual and needed } \\
\text { energy intake: } \\
\text { IG }=-228 \mathrm{kcal} / \text { day CG }=-783 \mathrm{kcal} / \text { day } \\
(p=0.0003)\end{array}$ \\
\hline \multirow{3}{*}{$\begin{array}{l}\text { Duncan [49] } \\
2006 \\
\text { UK }\end{array}$} & \multirow{3}{*}{$\begin{array}{l}\text { RCT } \\
\text { Effectiveness of dietetic } \\
\text { assistants (DAs) to reduce } \\
\text { in-hospital and } 4 \text { months } \\
\text { mortality. }\end{array}$} & \multirow{3}{*}{$\begin{array}{l}n 302(0 / 302) \\
\text { GT } 145 \\
\text { GC } 157 \\
83.5 \text { years } \\
\text { Follow-up: } \\
4 \text { months }\end{array}$} & \multirow{3}{*}{ NA } & \multirow{3}{*}{ Pathologic fracture } & $\begin{array}{l}\text { Mortality } \\
\text { In trauma unit IG } 4 \%, \text { CG } 10 \%(p=0.048) \\
\text { At } 4 \text { months IG } 13 \% \text {, CG } 23 \%(p=0.036)\end{array}$ \\
\hline & & & & & $\begin{array}{ll}\text { - } & \text { Energy intake = IG 1105; CG } \\
756 \mathrm{kcal} / \text { day }(p<0.001) \\
\text { Supplement intake: IG 409; } \\
\text { CG123 kcal/day }(p<0.001) \\
\text { MAC change: IG }-0.9 ; \text { CG }-1.3 \mathrm{~cm} \\
(p=0.002)\end{array}$ \\
\hline & & & & & $\begin{array}{l}\text { Weight change: IG }-0.35 ; \mathrm{CG}-1 \mathrm{~kg} \\
(p=0.16)\end{array}$ \\
\hline $\begin{array}{l}\text { Hommel [39] } \\
2007 \\
\text { Sweden }\end{array}$ & $\begin{array}{l}\text { Quasi-experimental } \\
\text { Effects of an improved care } \\
\text { intervention in relation to } \\
\text { nutritional status and } \\
\text { pressure ulcers }\end{array}$ & $\begin{array}{l}n 420 \\
\text { IG } 210(70 / 140) \\
\text { CG } 210(62 / 148) \\
81 \pm 10.4 \text { years }\end{array}$ & $\begin{array}{l}24.3 \pm 4.4 \mathrm{~kg} / \mathrm{m}^{2} \\
\text { MAC } 27.7 \pm 4.4 \mathrm{~cm} \\
\text { TSF } 14.8 \pm 6.8 \mathrm{~mm}\end{array}$ & NA & $\begin{array}{l}\text { Length of hospital stay: IG } 11.8 \pm 7.4 \text { vs. CG } \\
10.8 \pm 5.8 \text { days } \\
\text { Pressure ulcers: IG } 10 \% \text {; CG } 20.5 \%(p=0.009)\end{array}$ \\
\hline $\begin{array}{l}\text { Botella-Carretero } \\
{[24]} \\
2010 \\
\text { Spain }\end{array}$ & $\begin{array}{l}\text { RCT } \\
\text { Effect of perioperative } \\
\text { supplements on nutritional } \\
\text { status and postop } \\
\text { complications }\end{array}$ & $\begin{array}{l}n 60(16 / 44) \\
\text { IG } 30(6 / 24) \\
\text { CG } 30(10 / 20) \\
83.6 \pm 5.8 \text { years }\end{array}$ & $\begin{array}{l}24.4 \pm 3.1 \mathrm{~kg} / \mathrm{m}^{2} \\
\text { TSF } 11.9 \pm 4.1 \mathrm{~mm} \\
\text { MAC } 24.4 \pm 3.2 \mathrm{~cm} \\
\text { MNA } 18.6 \pm 3.4 \\
\text { Albumin } 33 \pm 4 \mathrm{~g} / \mathrm{L}\end{array}$ & $\begin{array}{l}\text { Weight loss }>5 \% \text { in } 1 \text { month or weight loss }> \\
10 \% \text { in } 6 \text { months, albumin }<27 \mathrm{~g} / \mathrm{L} \text {, renal } \\
\text { failure, hepatic insufficiency, respiratory } \\
\text { failure, and any gastrointestinal condition, any } \\
\text { nutritional support in the past } 6 \text { months }\end{array}$ & $\begin{array}{l}\text { CG: decrease and worse recovery of albumin } \\
\text { and prealbumin }(p=0.002 ; p=0.001) \\
\text { IG: } \uparrow \text { energy and protein intake }(p=0.042 ; \\
p<0.001) \\
\uparrow \text { protein intake } \rightarrow \downarrow \text { post-operative } \\
\text { complications OR }=0.925(0.869-0.985) \\
(p=0.003)\end{array}$ \\
\hline
\end{tabular}


Table 6. Cont

\begin{tabular}{|c|c|c|c|c|c|}
\hline $\begin{array}{l}\text { Author } \\
\text { Year } \\
\text { Origin }\end{array}$ & $\begin{array}{l}\text { Design } \\
\text { Aim }\end{array}$ & $\begin{array}{l}n \text { (Male/Female) } \\
\text { Age, Mean } \pm \text { SD } \\
\text { (Years) } \\
\text { Follow-Up (FU) }\end{array}$ & $\begin{array}{l}\text { BMI kg/m² } \\
(\text { Mean } \pm \text { SD) } \\
\text { Measurement of Body } \\
\text { Composition }\end{array}$ & Exclusion Criteria & Results \\
\hline $\begin{array}{l}\text { Fabian [23] } \\
2011 \\
\text { Austria }\end{array}$ & $\begin{array}{l}\text { RCT } \\
\text { Effect of nutritional } \\
\text { supplement on post-operative } \\
\text { oxidative stress and length of } \\
\text { hospital stay }\end{array}$ & $\begin{array}{l}n 23(0 / 23) \\
\text { IG } 14 \\
\text { CG } 9 \\
83.8 \pm 7.4 \text { years } \\
\text { Follow-up: } 3 \\
\text { weeks }\end{array}$ & $\begin{array}{l}21.2 \pm 3.4 \mathrm{~kg} / \mathrm{m}^{2} \\
\text { Albumin } 36.6 \pm 3.8 \mathrm{~g} / \mathrm{L}\end{array}$ & $\begin{array}{l}\text { Renal disease, liver failure, severe congestive } \\
\text { heart failure, severe pulmonary disease, } \\
\text { and any gastrointestinal condition that might } \\
\text { preclude the patient from adequate oral } \\
\text { nutritional intake }\end{array}$ & $\begin{array}{l}\text { IG } \uparrow \text { energy and protein intake }(p<0.05) \\
\text { Albumin, total protein, and total antioxidant } \\
\text { capacity (post-operative): } \\
\downarrow C G(p<0.05) \downarrow \text { IG } \\
\text { Advance oxidation protein products and } \\
\text { malondialdehyde: in CG levels still elevated } \\
\text { during time but not in IG } \\
\text { Length of hospital stay: IG } 17 \pm 4 \text { vs. CG } 19 \\
\pm 9 \text { days } \\
\text { Albumin } \leftrightarrow \text { CRP and total antioxidant } \\
\text { capacity }(p<0.05) \\
\text { Length of hospital stay } \leftrightarrow \text { AOPP and MDA } \\
(p<0.01)\end{array}$ \\
\hline $\begin{array}{l}\text { Hoekstra [25] } \\
2011 \\
\text { The Netherlands }\end{array}$ & $\begin{array}{l}\text { Prospective } \\
\text { Effectiveness of } \\
\text { a multidisciplinary } \\
\text { intervention on nutritional } \\
\text { status }\end{array}$ & $\begin{array}{l}n 127(31 / 96) \\
\text { IG } 61 \\
\text { CG } 66 \\
80.3 \pm 8.3 \text { years }\end{array}$ & $26.8 \pm 4.5 \mathrm{~kg} / \mathrm{m}^{2}$ & $\begin{array}{l}\text { Severe dementia, cancer, pathologic fracture, } \\
\text { renal and hepatic dysfunction, pacemaker }\end{array}$ & $\begin{array}{l}\text { IG } \uparrow \text { energy intake protein, vitamin D, zinc, } \\
\text { calcium }(p<0.01) \\
\text { IG lower reduction of EuroQol-5D }(p<0.05) \\
\downarrow^{*} \text { BMI, BCM, and FM ( } 3 \text { months) (both } \\
\text { groups) }\end{array}$ \\
\hline $\begin{array}{l}\text { Li [28] } \\
2013 \\
\text { Taiwan }\end{array}$ & $\begin{array}{l}\text { Randomized ( } 1 \text { year) } \\
\text { Effects of protein-energy } \\
\text { malnutrition on the } \\
\text { functional recovery }\end{array}$ & $\begin{array}{l}n 162(51 / 111) \\
\text { IG } 80 \\
\text { CG } 82 \\
78.2 \text { years }\end{array}$ & NA & Cognitive impairment, terminally ill & $\begin{array}{l}\text { Malnutrition prevalence: IG } 60 \% \text { vs. CG } 67 \% \\
\text { MN } \rightarrow \downarrow \text { performance of ADL, IADL, } \\
\text { and recovery of walking ability }(p<0.05) \\
\text { IG } \rightarrow \uparrow \text { performance of ADL, IADL, } \\
\text { and recovery of walking ability }(p<0.01)\end{array}$ \\
\hline $\begin{array}{l}\text { Wyers [29] } \\
2013 \\
\text { The Netherlands }\end{array}$ & $\begin{array}{l}\text { RCT } \\
\text { Cost-effectiveness of dietary } \\
\text { intervention comprising } \\
\text { combined dietetic counseling } \\
\text { and ONS }\end{array}$ & $\begin{array}{l}n 152(108 / 44) \\
\text { IG } 73 \\
\text { CG } 79 \\
78.5 \text { years }\end{array}$ & NA & $\begin{array}{l}\text { Pathological or periprosthetic fracture, disease } \\
\text { of bone metabolism, life expectancy }<1 \text { year, } \\
\text { ONS before hospital admission, dementia. }\end{array}$ & $\begin{array}{l}\text { The additional cost of the nutritional } \\
\text { intervention was only } 3 \% \text { of the total cost } \\
\text { Total cost was not significantly different } \\
\text { between both groups } \\
\text { Nutritional intervention was likely to be cost } \\
\text { effective for weight as the outcome over } \\
3 \text { months }\end{array}$ \\
\hline $\begin{array}{l}\text { Myint [52] } \\
2013 \\
\text { Hong Kong }\end{array}$ & $\begin{array}{l}\text { RCT } \\
\text { Clinical, nutritional and } \\
\text { rehabilitation effects of an } \\
\text { oral nutritional } \\
\text { supplementation }\end{array}$ & $\begin{array}{l}n 121(41 / 80) \\
\text { IG } 61 \\
\text { CG } 60 \\
81.3 \pm 6.5 \text { years } \\
\text { Follow-up: } \\
6 \text { months }\end{array}$ & $\begin{array}{l}20.7 \pm 2.9 \mathrm{~kg} / \mathrm{m}^{2} \\
\text { TSF } 12.6 \pm 5.6 \mathrm{~mm} \\
\text { MAC } 24.3 \pm 3 \mathrm{~cm} \\
\text { Albumin } 29.3 \pm 4.6 \mathrm{~g} / \mathrm{L}\end{array}$ & $\begin{array}{l}\text { Tube feeding, unstable medical condition, BMI } \\
\geq 25 \mathrm{~kg} / \mathrm{m}^{2} \text {, malignancy, contraindication for } \\
\text { high-protein diet, and mentally incapacitated }\end{array}$ & $\begin{array}{l}\text { BMI decrease of } 0.25 \text { and } 0.003 \mathrm{~kg} / \mathrm{m}^{2} \text { in the } \\
\text { ONS group, and } 0.72 \text { and } 0.49 \mathrm{~kg} / \mathrm{m}^{2} \text { at } \\
\text { hospital and follow-up }(p=0.012) \\
\text { Length of hospital stay was shortened by } 3.8 \\
\text { days in the ONS group ( } p=0.04) \\
\text { Intake adequate: } 67 \% \text { in the ONS group, } 9 \% \\
\text { in the control group ( } p<0.001)\end{array}$ \\
\hline
\end{tabular}


Table 6. Cont

\begin{tabular}{|c|c|c|c|c|c|}
\hline $\begin{array}{l}\text { Author } \\
\text { Year } \\
\text { Origin }\end{array}$ & $\begin{array}{l}\text { Design } \\
\text { Aim }\end{array}$ & $\begin{array}{l}n \text { (Male/Female) } \\
\text { Age, Mean } \pm \text { SD } \\
\text { (Years) } \\
\text { Follow-Up (FU) }\end{array}$ & $\begin{array}{l}\mathrm{BMI} \mathrm{kg} / \mathrm{m}^{2} \\
(\text { Mean } \pm \mathrm{SD}) \\
\text { Measurement of Body } \\
\text { Composition }\end{array}$ & Exclusion Criteria & Results \\
\hline $\begin{array}{l}\text { Anbar [17] } \\
2014 \\
\text { Israel }\end{array}$ & $\begin{array}{l}\text { RCT } \\
\text { Optimization of } \\
\text { supplementation by } \\
\text { measurement of resting } \\
\text { energy requirements and the } \\
\text { effect on outcomes }\end{array}$ & $\begin{array}{l}n 50(17 / 33) \\
\text { IG } 22 \\
\text { CG } 28 \\
83.1 \pm 6.3 \text { years }\end{array}$ & $24.9 \pm 3.9 \mathrm{~kg} / \mathrm{m}^{2}$ & $\begin{array}{l}\text { Presented to hospital }>48 \mathrm{~h} \text { after the injury, } \\
\text { steroids and/or immunosuppression therapy, } \\
\text { oncologic disease, multiple fractures, dementia }\end{array}$ & $\begin{array}{l}\text { ONS }=19.6 \% \text { of total energy } \\
\text { IG: } \\
\uparrow \text { Energy and protein intake }(p=0.001) \\
\downarrow \text { complications }(p=0.012) \text { and infections } \\
(p=0.008) \\
\downarrow \text { length of hospital stay }(p=0.061) \\
\text { In all patients: } \\
\text { Energy balance } \leftrightarrow \text { complications }(\mathrm{r}=-0.417 \\
p=0.003) \text { and with length of hospital stay } \\
(\mathrm{r}=-0.282 ; p=0.049)\end{array}$ \\
\hline $\begin{array}{l}\text { Ekinci [55] } \\
2016 \\
\text { Turkey }\end{array}$ & $\begin{array}{l}\text { RCT } \\
\text { Effects of CaHMB on wound } \\
\text { healing, mobilization, fat-free } \\
\text { mass and muscle strength }\end{array}$ & $\begin{array}{l}n 62(0 / 62) \\
\text { IG } 32 \\
\text { CG } 30 \\
82.6 \pm 7.1 \text { years }\end{array}$ & $22.0 \pm 2.4 \mathrm{~kg} / \mathrm{m}^{2}$ & $\begin{array}{l}\text { Diabetes, renal and hepatic failure, } \\
\text { gastrointestinal intolerance, endocrine } \\
\text { pathology, and dementia. }\end{array}$ & $\begin{array}{l}\text { Patients who were mobile on day } 30 \text { : } \\
\text { - IG } 81.3 \% \text { vs. CG } 26.7 \%(p=0.001) \\
\text { Muscle strength on day } 30 \text { was higher in IG } \\
\text { vs. CG }(p=0.026)\end{array}$ \\
\hline $\begin{array}{l}\text { Malafarina [54] } \\
2017 \\
\text { Spain }\end{array}$ & $\begin{array}{l}\text { RCT } \\
\text { Effects of ONS on muscle } \\
\text { mass and nutritional } \\
\text { biomarkers }\end{array}$ & $\begin{array}{l}n 107 \\
\text { IG } 55 \\
\text { CG } 52 \\
85.4 \pm 6.3 \text { years }\end{array}$ & $\begin{array}{l}25.4 \pm 4.9 \mathrm{~kg} / \mathrm{m}^{2} \\
\text { Albumin } 3.1 \pm 0.4 \mathrm{~g} / \mathrm{dL}\end{array}$ & $\begin{array}{l}\text { Diabetes, Barthel index }<40 \text { prior to the } \\
\text { fracture, tumor, pathological or high-impact } \\
\text { fractures }\end{array}$ & $\begin{array}{l}\text { BMI and ALM was stable in IG, but } \\
\text { decreased in CG. } \\
\text { ONS }(p=0.006) \text {, function ambulation } \\
\text { categories prior to the fracture }(p=0.007) \\
\text { and Barthel index prior to the fracture } \\
(p=0.007) \text { are protective for loss of ALM }\end{array}$ \\
\hline
\end{tabular}


Table 7. Characteristics of the nutritional intervention and composition of the nutritional supplementation used in the included studies.

\begin{tabular}{|c|c|c|c|c|c|}
\hline $\begin{array}{l}\text { Author } \\
\text { Year } \\
\text { Origin }\end{array}$ & $\begin{array}{l}\text { Type of Supplement } \\
\text { Administration Method }\end{array}$ & kcal & Nutritional Composition & $\begin{array}{l}\text { Treatment Duration } \\
\text { Adherence Rate (\%) }\end{array}$ & Control Group \\
\hline $\begin{array}{l}\text { Schürch [50] } \\
\quad 1998 \\
\text { Switzerland }\end{array}$ & $\begin{array}{l}\text { Oral liquid supplement; single oral } \\
\text { dose of vit } D_{3} 200.000 \text { UI } \\
\text { Ca: } 550 \mathrm{mg} \text { /day }\end{array}$ & $250 \mathrm{kcal} /$ day & $\begin{array}{l}20 \text { g protein, } 3.1 \mathrm{~g} \text { lipid, } \\
35.7 \text { g carbohydrates, } \\
90 \% \text { milk proteins }\end{array}$ & $\begin{array}{c}5 \text { days a week for } 6 \text { months } \\
\text { AR: } \\
\text { IG } 73 \% \\
\text { CG } 80 \%\end{array}$ & $\begin{array}{c}\text { Placebo: } 54.5 \text { g carbohydrates } \\
\text { Single oral dose of vitamin D } \\
200.000 \text { UI } \\
\text { Calcium: } 550 \mathrm{mg} / \text { day }\end{array}$ \\
\hline $\begin{array}{l}\text { Espaulella [36] } \\
2000 \\
\text { Spain }\end{array}$ & $\begin{array}{l}\text { Oral liquid supplement } \\
200 \mathrm{~mL}\end{array}$ & $149 \mathrm{kcal}$ & $\begin{array}{l}20 \mathrm{~g} \text { protein, } 800 \mathrm{mg} \\
\text { calcium, } 25 \mathrm{IU} \text { vitamin D3 }\end{array}$ & $\begin{array}{l}60 \text { days } \\
\text { AR: } \\
\text { IG } 94.1 \% \\
\text { CG } 94.2 \%\end{array}$ & $\begin{array}{l}\text { Placebo } 200 \mathrm{~mL}, 155 \mathrm{kcal} ; \\
\text { mainly carbohydrates }\end{array}$ \\
\hline $\begin{array}{l}\text { Bruce [51] } \\
2003 \\
\text { Australia }\end{array}$ & $\begin{array}{l}\text { Oral liquid supplement } \\
\text { (235 mL/day) }\end{array}$ & $352 \mathrm{kcal}$ & $\begin{array}{l}17.6 \mathrm{~g} \text { protein, } 11.8 \mathrm{~g} \text { fat, } \\
44.2 \mathrm{~g} \text { carbohydrate, } \\
\text { vitamins and minerals }\end{array}$ & 28 days after surgery & Hospital diet only \\
\hline $\begin{array}{l}\text { Houwing [56] } \\
2003 \\
\text { The Netherlands }\end{array}$ & $\begin{array}{l}\text { Oral liquid supplement } \\
\quad(400 \mathrm{~mL} / \text { day })\end{array}$ & $500 \mathrm{kcal}$ & $40 \mathrm{~g}$ protein & $\begin{array}{l}\text { Immediately postoperatively } \\
\text { during } 4 \text { weeks or until } \\
\text { discharge } \\
\text { AR: } 75 \% \text { of patients consumed } \\
>75 \% \text { of daily dose }\end{array}$ & $\begin{array}{l}\text { Non-caloric placebo } \\
\text { supplement }\end{array}$ \\
\hline $\begin{array}{l}\text { Sullivan [48] } \\
2004 \\
\text { USA }\end{array}$ & $\begin{array}{l}\text { Standard care + post-operative } \\
\text { nightly via enteral feeding tube: } \\
1375 \mathrm{~mL}(125 \mathrm{~mL} / \mathrm{h}) \text { over } 11 \mathrm{~h}\end{array}$ & $1031 \mathrm{kcal}$ & $85.8 \mathrm{~g}$ protein & $\begin{array}{c}\text { When volitional intake } \\
\text { exceeded } 90 \% \text { of estimated } \\
\text { requirements for } 3 \text { consecutive } \\
\text { days or was discharged: mean } \\
15.8 \pm 16.4 \text { days } \\
\text { AR: } 83.3 \%\end{array}$ & Standard care \\
\hline $\begin{array}{l}\text { Tidermark [53] } \\
\text { 2004 } \\
\text { Sweden }\end{array}$ & $\begin{array}{l}\text { PR: protein-rich liquid supplement } \\
\quad(200 \mathrm{~mL} / \text { day) } \\
\text { PR-N: PR + nandrolone decanoate } \\
\text { injections (every third week) } \\
1 \mathrm{~g} \text { of calcium + } 400 \text { IE vitamin D3 }\end{array}$ & $\begin{array}{c}200 \mathrm{kcal} / \text { day } \\
\text { nandrolone: } 25 \mathrm{mg} \\
\text { intramuscular injection }\end{array}$ & $20 \mathrm{~g}$ protein & 6 months & $\begin{array}{c}\text { Standard treatment } \\
1 \mathrm{~g} \text { of calcium }+400 \mathrm{IE} \text { vitamin } \\
\text { D3 }\end{array}$ \\
\hline $\begin{array}{l}\text { Eneroth [16] } \\
2005 \\
\text { Sweden }\end{array}$ & $\begin{array}{l}\text { Hospital diet }+ \text { intravenous } \\
\text { nutrition ( } 1 \text { l/day) followed by } \\
400 \mathrm{~mL} / \text { day oral supplement }\end{array}$ & $\begin{array}{l}\text { Oral supplement } \\
400 \mathrm{kcal} / \text { day }\end{array}$ & $\begin{array}{l}\text { IV: amino acids, fat, } \\
\text { carbohydrate, } \\
\text { and electrolytes }\end{array}$ & $\begin{array}{c}3 \text { days } \rightarrow \text { IV } \\
7 \text { days } \rightarrow \text { oral }\end{array}$ & Hospital diet only \\
\hline $\begin{array}{l}\text { Duncan [49] } \\
2006 \\
\text { UK }\end{array}$ & NA & $\begin{array}{l}\text { Mean supplement: } \\
409 \mathrm{kcal} / \text { day }\end{array}$ & NA & NA & $\begin{array}{l}\text { Mean standard supplement: } \\
123 \mathrm{kcal} / \text { day }\end{array}$ \\
\hline
\end{tabular}


Table 7. Cont.

\begin{tabular}{|c|c|c|c|c|c|}
\hline $\begin{array}{l}\text { Author } \\
\text { Year } \\
\text { Origin }\end{array}$ & $\begin{array}{c}\text { Type of Supplement } \\
\text { Administration Method }\end{array}$ & kcal & Nutritional Composition & $\begin{array}{l}\text { Treatment Duration } \\
\text { Adherence Rate (\%) }\end{array}$ & Control Group \\
\hline $\begin{array}{l}\text { Hommel [39] } \\
2007 \\
\text { Sweden }\end{array}$ & $\begin{array}{l}\text { Oral nutritional supplement twice } \\
\text { a day }\end{array}$ & $\begin{array}{l}125 \mathrm{kcal} / 100 \mathrm{~mL} \text { enriched } \\
\text { with arginine, zinc, } \\
\text { vitamins A, B, C, and } \mathrm{E}, \\
\text { selenium, and carotenoids }\end{array}$ & NA & From post-surgery to discharge & NA \\
\hline $\begin{array}{c}\text { Botella-Carretero } \\
{[24]} \\
2010 \\
\text { Spain }\end{array}$ & $\begin{array}{l}\text { Oral nutritional supplement intake } \\
\qquad(2 \times 200 \mathrm{~mL} / \text { day })\end{array}$ & $400 \mathrm{kcal} /$ day & $40 \mathrm{~g}$ protein/day & $\begin{array}{l}\text { From admission until discharge } \\
\text { AR } 52.2 \pm 12.1 \%\end{array}$ & Control group: no supplement \\
\hline $\begin{array}{c}\text { Fabian [23] } \\
2011 \\
\text { Austria }\end{array}$ & Oral liquid supplement & $\begin{array}{c}\text { Supplements were } \\
\text { administered when intake } \\
\text { of energy }<20 \mathrm{kcal} \\
\text { and } / \text { or protein }<1 \mathrm{~g} / \mathrm{kg} \\
\text { body weight } / \text { day }\end{array}$ & $\begin{array}{l}40 \% \text { protein, } 41 \% \\
\text { carbohydrate, } 19 \% \text { fat, } \\
\text { vitamins and minerals }\end{array}$ & From post-surgery to discharge & Standard medical treatment \\
\hline $\begin{array}{c}\text { Hoekstra [25] } \\
2011 \\
\text { The Netherlands }\end{array}$ & $\begin{array}{c}\text { Nurse and doctor encouraged and } \\
\text { motivated patients to eat and drink; } \\
\text { if MNA }<24 \text {, dietician consulted } \\
\text { with the patient }\end{array}$ & NA & NA & NA & Standard nutritional care \\
\hline $\begin{array}{c}\text { Wyers [29] } \\
2013 \\
\text { The Netherlands }\end{array}$ & $\begin{array}{l}\text { Oral liquid nutritional supplement } \\
(500 \mathrm{~mL} / \text { day }) \\
\text { Dietetic counseling }\end{array}$ & $500 \mathrm{kcal}$ & $40 \mathrm{~g}$ protein & $\begin{array}{l}\text { Started during hospital } \\
\text { admission and continued until } \\
3 \text { months after surgery }\end{array}$ & $\begin{array}{c}\text { Usual care } \\
\text { ONS on demand: } 13 \% \text { received } \\
\text { ONS and } 23 \% \text { received dietetic } \\
\text { counseling }\end{array}$ \\
\hline $\begin{array}{l}\text { Myint [52] } \\
2013 \\
\text { Hong Kong }\end{array}$ & $\begin{array}{c}\text { Oral liquid nutritional supplement } \\
\text { (240 mL twice daily) } \\
1.2 \mathrm{~g} \text { of calcium }+800-1000 \mathrm{IU} \\
\text { vitamin D }\end{array}$ & $500 \mathrm{kcal}$ & $18-24$ g protein & $\begin{array}{c}\text { Started within } 3 \text { days after } \\
\text { admission until discharge or } 28 \\
\text { days } \\
\text { AR }=77.7 \%\end{array}$ & $\begin{array}{c}\mathrm{NA} \\
1.2 \mathrm{~g} \text { of calcium }+800-1000 \mathrm{IU} \\
\text { vitamin D }\end{array}$ \\
\hline $\begin{array}{l}\text { Anbar [17] } \\
2014 \\
\text { Israel }\end{array}$ & $\begin{array}{c}\text { Standard ONS }(237 \mathrm{~mL}) \text { or } \\
\text { diabetic ONS }(237 \mathrm{~mL}) \\
\text { Patients received the difference } \\
\text { between intake and measured } \\
\text { energy expenditure }\end{array}$ & $\begin{array}{l}355 \mathrm{kcal} \\
237 \mathrm{kcal}\end{array}$ & $\begin{array}{l}13.5 \mathrm{~g} \text { protein } \\
9.9 \mathrm{~g} \text { protein }\end{array}$ & $\begin{array}{l}\text { Started } 24 \mathrm{~h} \text { after surgery } \\
\qquad \mathrm{AR}=100 \%\end{array}$ & $\begin{array}{l}\text { Usual hospital diet }=1800 \mathrm{kcal}, \\
80 \mathrm{~g} \text { protein }\end{array}$ \\
\hline
\end{tabular}


Table 7. Cont.

\begin{tabular}{|c|c|c|c|c|c|}
\hline $\begin{array}{l}\text { Author } \\
\text { Year } \\
\text { Origin }\end{array}$ & $\begin{array}{l}\text { Type of Supplement } \\
\text { Administration Method }\end{array}$ & kcal & Nutritional Composition & $\begin{array}{l}\text { Treatment Duration } \\
\text { Adherence Rate (\%) }\end{array}$ & Control Group \\
\hline $\begin{array}{l}\text { Ekinci [55] } \\
\text { 2016Turkey }\end{array}$ & $\begin{array}{l}\text { Oral liquid nutritional supplement } \\
\text { (220 mL twice daily) }\end{array}$ & NA & $\begin{array}{c}36 \mathrm{~g} \text { protein } \\
3 \mathrm{~g} \text { CaHMB } \\
1000 \text { IU vitamin D }\end{array}$ & 30 days & $\begin{array}{c}\text { Usual hospital diet: } 1900 \mathrm{kcal} \text {, } \\
76 \mathrm{~g} \text { protein, } 63 \mathrm{~g} \text { fat }\end{array}$ \\
\hline $\begin{array}{c}\text { Malafarina [54] } \\
2017 \\
\text { Spain }\end{array}$ & $\begin{array}{l}\text { Oral liquid nutritional supplement } \\
\text { (220 mL twice daily) }\end{array}$ & $660 \mathrm{kcal}$ & $\begin{array}{c}60 \mathrm{~g} \text { protein } \\
4.6 \mathrm{~g} \text { CaHMB } \\
1500 \mathrm{IU} \text { vitamin D }\end{array}$ & $\begin{array}{c}\text { During hospital admission, } \\
\text { until discharge } \\
\text { Mean treatment duration: } 42.3 \\
\quad \pm 20.9 \text { days } \\
\text { AR = all of the subjects took } \\
\text { more than } 80 \%\end{array}$ & $\begin{array}{c}\text { Usual hospital diet: } 1500 \mathrm{kcal} \text {, } \\
87 \mathrm{~g} \text { protein, } 59 \mathrm{~g} \text { fat }\end{array}$ \\
\hline
\end{tabular}




\section{Discussion}

Malnutrition is a subject under intense discussion in geriatric research [57-59], as it is very prevalent in older people with hip fracture, it negatively influences functional recovery after fracture, it increases healthcare spending, and it is associated with high mortality. It appears that nutritional intervention aids the prevention of complications in geriatric patients with hip fracture. This review is an attempt to summarize existing evidence of these aspects. To our knowledge, this is the first review to assess the nutritional status of older people with hip fracture and how it influences complications and mortality.

Despite the variability in the main objective of the included studies, the results are homogeneous in the evidence that subjects with hip fracture have anthropometric indices indicative of malnutrition (Table 2). In addition, there is evidence that subjects with worse nutritional status have more complications (Table 3) and increased mortality (Table 4). There is a lot of variability in the main objective, as well as in the type of nutritional intervention (dietary advice, use of nutritional supplements) and in the amount of calories used in the included studies (Table 7). Despite this variability in the methods used in the studies, overall nutritional intervention has been shown to reduce complications and avoid weight loss in elderly subjects with hip fracture (Table 6).

The prevalence of malnutrition in older patients with hip fracture is higher than in community-dwelling older adults $[60,61]$. A further problem was associated with an increase in calorie expenditure, secondary to systemic inflammatory response, without a corresponding intake increase, whereby nutritional intake remained smaller than requirements due to factors such as pain, being bedridden, and reduced mobility [25,49].

A reduction in intake is often observed in older people, causing it to be lower than requirements [57]. These changes in intake have a multi-factor origin, among which the most frequent factors are alterations in sensory organs, loss of teeth, lack of a principal caregiver, and, in some cases, the adverse effects of certain drugs [62]. These intake alterations constitute a well-known geriatric syndrome defined as anorexia of ageing [63]. Calorie and/or protein deficits can contribute to the pathophysiology of fractures, especially through two mechanisms: (1) loss of strength and muscle mass (sarcopenia), which increases the risk of falls; and (2) low bone mineral density (osteoporosis), which reduces the resistance of bones to trauma, increasing the risk of fracture [1].

The observed variability in the parameters of nutritional status in hip fracture patients could be due to the lack of a universal consensus as to the best measure to diagnose protein-energy malnutrition. This lack of universality limits our comparison of the various studies, also making it difficult to carry out a consistent malnutrition diagnosis, which, in certain cases, can delay the clinical decision to prescribe nutritional treatment for these patients.

Despite this, the observed trend is uniform and shows that malnourished older people are at a greater risk of fracture and that the prevalence of malnutrition is high in geriatric patients admitted with hip fracture. Patients with intracapsular fracture usually have low BMI, while patients with trochanteric fracture tend to have high BMI $[20,64]$. Low BMI is associated with protein deficit (type II nutrients, important for maintaining weight) and type I nutrients are important for bone metabolism. In relation to this, "BMI paradox" is valid in the elderly, in which an increase in fat mass and a decrease of muscle mass are observed, and for this reason falsely high values of BMI can mask the presence of sarcopenia [65]. Despite the important limitations of the prognostic meaning of BMI in the elderly, this remains a fundamental index to assess the nutritional status for its simplicity and repeatability, and most validated nutrition assessment tools include BMI. Recent articles have proposed that the normal cut-off considered by the World Health Organization (WHO) (18.5-20.0 kg/m²) should be modified with the values that have been shown to be associated with lower mortality in the elderly $\left(23-29 \mathrm{~kg} / \mathrm{m}^{2}\right)$ [66]. It would be advisable to complete the nutritional assessment by evaluating the body composition (with dual-energy absorptiometry (DXA) or with bioimpedance analysis) $[67,68]$. The problem is different if we consider the concentration of albumin for the diagnosis of malnutrition. The blood concentration of albumin may be a good nutritional index if the inflammatory state is taken 
into account, considering that its concentration does not depend only on nutritional status [40]. On the other hand, albumin has been shown to be a good prognostic index in hospitalized patients [69].

Screening tools such as the Mini-Nutritional Assessment Short-Form (MNA-SF) were able to diagnose a nutritional problem before it manifested through changes in the biochemical markers of malnutrition (such as albumin or total protein) [70]. Factors such as cognitive impairment and disability in the basic activities of daily living (ADL) were associated with lower scores in the MNA-SF [71]. This tool was also shown to be a predictive factor of destination upon discharge following the fracture [72]. Selective deficiencies such as lack of vitamin D are very prevalent in older people [73].

As well as the known effects of this deficiency on bone metabolism, there is a high concentration of vitamin D receptors in muscle tissue [7]. This situation could explain why the lack of this vitamin (scant diet input, little exposure to the sun, and the ability to make vitamin D within the skin declines with age) is so obviously associated with reduced muscle strength and a worse functional status, involving factors that increase the risk of fall and fracture [42].

The high prevalence of malnutrition in people with dementia could be one of the pathophysiological mechanisms for the high risk of falls and fractures, as well as for their poor functional recovery after a fracture $[27,37,42,74]$. People with dementia suffered an increase in incidence of hip fracture, as dementia is a risk factor for hip fracture [75]. Strategies for the prevention of hip fractures are very important in people with dementia because they present a higher prevalence of complications, higher risk of institutionalization, and worse functional recovery [3]. Moreover, dementia is an independent predictor for mortality [76].

Malnutrition, which is very prevalent in geriatric patients with hip fracture, is associated with the incidence of complications, with length of hospital stay (and thus increase in cost), and with mortality. Hip fracture continues to be a pathology with high mortality. In spite of the achievement of a reduction in the incidence of hip fractures, mortality has not decreased [77]. Intra-hospital mortality of elderly patients with hip fracture $(7.4 \%)$ is comparable with mortality of elderly patients with heart failure $(8 \%)$ [78]. The problem is that hip fracture represents an acute potentially preventable disease, for example by implanting exercise programs that have been shown to reduce the risk of falling [79]. It will be necessary to improve the post-surgical treatment to reduce complications and mortality, which at 3 years is almost twice that of patients with heart failure [80,81].

Patients with hip fracture show a state of hypercatabolism secondary to reduced intake, loss of blood, and inflammation, which leads to a reduction in plasma proteins, which are important mechanisms for the defense of oxidative stress. Cell regeneration determines an increase in the production of free radicals at the site of the fracture. Plasma oxidant markers malondialdehyde (MDA) and advanced oxidation protein products (AOPP) were significantly positively, while albumin and total antioxidant capacity (TAC) are significantly negatively associated with the duration of hospitalization.

Several studies observed lower scores in nutritional indices, such as BMI, in geriatric patients who died following a hip fracture, as compared to those who lived [82]. It may be possible to reduce mortality with adequate nutritional intervention [49]. A difficult question to answer is whether nutritional supplementation is indicated for all patients with hip fracture or only for malnourished patients. Supplementation prevented weight loss in both malnourished and well-nourished patients. This association was directly related to the dose administered [51]. A higher protein intake was associated with a lower risk of post-surgery complications [24], and an adequate energy intake reduced the development of complications and was associated with a shorter duration of hospital stay [17].

Therefore, the results of this review support the indications of the European Society of Parenteral and Enteral Nutrition (ESPEN) guidelines, according to which all older adult patients with hip fracture should receive nutritional supplements during hospitalization [83]. 


\section{Conclusions}

The prevalence of malnutrition is very high in older people and increases further in older people with high comorbidities as well as in geriatric patients. Malnutrition is associated to functional alterations and this can be a cause as well as a consequence of fractures.

Malnutrition prevention could be associated with a reduction in the incidence of fractures, and with a better functional recovery following hip fracture. Fall prevention campaigns as well as advice on healthy and active ageing have contributed to the reduction in the incidence of hip fractures. The inclusion in care plans for geriatric patients with hip fracture of both nutritional assessments and the treatment of malnutrition could contribute to a better functional recovery and a reduction of mortality.

Supplementary Materials: The following are available online at http:/ / www.mdpi.com/2072-6643/10/5/555/s1, Table S1: Results of quality assessment of the observational included studies, Table S2: Results of quality assessment of included intervention studies.

Author Contributions: V.M. is the principal investigator, he conceived the study, and he wrote this paper. This research is part of V.M.'s PhD project. S.C. contributed to the paper search, data abstraction, and the redaction of the paper. J.Y.R., J.A.K., O.B., and J.A.M. helped in the critical analysis of the findings, and in both the correction and editing of this manuscript. M.A.Z. is the supervisor of the PhD project; she contributed to the critical analysis of findings and the writing and editing of this paper.

Conflicts of Interest: V.M., S.C., O.B., J.A.K., J.A.M., M.A.Z. declare no conflicts of interest with this article. J.-Y.R.: Consulting fees or paid advisory boards: IBSA-GENEVRIER, MYLAN, RADIUS HEALTH, PIERRE FABRE. Lecture fees when speaking at the invitation of sponsor: IBSA-GENEVRIER, MYLAN, CNIEL, DAIRY RESEARCH COUNCIL (DRC). Grant Support from Industry (all through Institution): IBSA-GENEVRIER, MYLAN, CNIEL, RADIUS HEALTH.

\section{References}

1. Huang, Z.; Himes, J.H.; McGovem, P.G. Nutrition and subsequent fracture risk among a national cohort of white women. Am. J. Epidemiol. 1996, 144, 124-134. [CrossRef] [PubMed]

2. Peeters, C.M.M.; Visser, E.; Van De Ree, C.L.P.; Gosens, T.; Den Oudsten, B.L.; De Vries, J. Quality of life after hip fracture in the elderly: A systematic literature review. Injury 2016, 47, 1369-1382. [CrossRef] [PubMed]

3. Uriz-Otano, F.; Uriz-Otano, J.I.; Malafarina, V. Factors associated with short-term functional recovery in elderly people with a hip fracture. Influence of cognitive impairment. J. Am. Med. Dir. Assoc. 2015, 16, 215-220. [CrossRef] [PubMed]

4. NICE. The National Institute for Health. Hip Fracture in Adults. Available online: https:/ /www.nice.org. uk/guidance/qs16 (accessed on 10 December 2017).

5. Bellelli, G.; Mazzola, P.; Corsi, M.; Zambon, A.; Corrao, G.; Castoldi, G.; Zatti, G.; Annoni, G. The combined effect of ADL impairment and delay in time from fracture to surgery on 12-month mortality: An observational study in orthogeriatric patients. J. Am. Med. Dir. Assoc. 2012, 13, 664.e9-664.e14. [CrossRef] [PubMed]

6. Leal, J.; Gray, A.M.; Prieto-Alhambra, D.; Arden, N.K.; Cooper, C.; Javaid, M.K.; Judge, A. Impact of hip fracture on hospital care costs: A population-based study. Osteoporos. Int. 2016, 27, 549-558. [CrossRef] [PubMed]

7. Pioli, G.; Lauretani, F.; Pellicciotti, F.; Pignedoli, P.; Bendini, C.; Davoli, M.L.; Martini, E.; Zagatti, A.; Giordano, A.; Nardelli, A.; et al. Modifiable and non-modifiable risk factors affecting walking recovery after hip fracture. Osteoporos. Int. 2016, 27, 2009-2016. [CrossRef] [PubMed]

8. Wiklund, R.; Toots, A.; Conradsson, M.; Olofsson, B.; Holmberg, H.; Rosendahl, E.; Gustafson, Y.; Littbrand, H. Risk factors for hip fracture in very old people: A population-based study. Osteoporos. Int. 2016, 27, $923-931$. [CrossRef] [PubMed]

9. Torres, M.J.; Féart, C.; Samieri, C.; Dorigny, B.; Luiking, Y.; Berr, C.; Barberger-Gateau, P.; Letenneur, L. Poor nutritional status is associated with a higher risk of falling and fracture in elderly people living at home in France: The Three-City cohort study. Osteoporos. Int. 2015, 26, 2157-2164. [CrossRef] [PubMed]

10. Hedstrom, M.; Ljungqvist, O.; Cederholm, T. Metabolism and catabolism in hip fracture patients: Nutritional and anabolic intervention-A review. Acta Orthop. 2006, 77, 741-747. [CrossRef] [PubMed] 
11. National Heart Lung and Blood Institute. Study Quality Assessment Tools. Available online: https://www. nhlbi.nih.gov/health-topics/study-quality-assessment-tools (accessed on 10 December 2017).

12. Lumbers, M.; New, S.A.; Gibson, S.; Murphy, M.C. Nutritional status in elderly female hip fracture patients: Comparison with an age-matched home living group attending day centres. Br. J. Nutr. 2001, 85, 733-740. [CrossRef] [PubMed]

13. Durillo, F.T.P.; Durántez, J.T.; Villar, A.B.V.; Vico, A.B.S.; Camarero, M.D.M.C.; Durillo, J.P. Estudio comparativo de la ingesta alimentaria y el estado nutricional en ancianas con y sin fractura de cadera. Atención Primaria 2011, 43, 362-368. [CrossRef] [PubMed]

14. Nematy, M.; Hickson, M.; Brynes, A.E.; Ruxton, C.H.S.; Frost, G.S. Vulnerable patients with a fractured neck of femur: Nutritional status and support in hospital. J. Hum. Nutr. Diet. 2006, 19, 209-218. [CrossRef] [PubMed]

15. Murphy, M.C.; Brooks, C.N.; New, S.A.; Lumbers, M.L. The use of the Mini-Nutritional Assessment (MNA) tool in elderly orthopaedic patients. Eur. J. Clin. Nutr. 2000. [CrossRef]

16. Eneroth, M.; Olsson, U.B.; Thorngren, K.G. Insufficient fluid and energy intake in hospitalised patients with hip fracture. A prospective randomised study of 80 patients. Clin. Nutr. 2005, 24, 297-303. [CrossRef] [PubMed]

17. Anbar, R.; Beloosesky, Y.; Cohen, J.; Madar, Z.; Weiss, A.; Theilla, M.; Koren Hakim, T.; Frishman, S.; Singer, P. Tight Calorie Control in geriatric patients following hip fracture decreases complications: A randomized, controlled study. Clin. Nutr. 2014, 33, 23-28. [CrossRef] [PubMed]

18. Koren-Hakim, T.; Weiss, A.; Hershkovitz, A.; Otzrateni, I.; Grosman, B.; Frishman, S.; Salai, M.; Beloosesky, Y. The relationship between nutritional status of hip fracture operated elderly patients and their functioning, comorbidity and outcome. Clin. Nutr. 2012, 31, 917-921. [CrossRef] [PubMed]

19. Pérez Durillo, F.T.; Ruiz López, M.D.; Bouzas, P.R.; Martín-Lagos, Y.A. Estado nutricional en ancianos con fractura de cadera. Nutr. Hosp. 2010, 25, 676-681. [CrossRef] [PubMed]

20. Maffulli, N.; Dougall, T.W.; Brown, M.T.; Golden, M.H. Nutritional differences in patients with proximal femoral fractures. Age Ageing 1999, 28, 458-462. [CrossRef] [PubMed]

21. Bohl, D.D.; Shen, M.R.; Hannon, C.P.; Fillingham, Y.A.; Darrith, B.; Della Valle, C.J. Serum Albumin Predicts Survival and Postoperative Course Following Surgery for Geriatric Hip Fracture. J. Bone Joint Surg. Am. 2017, 99, 2110-2118. [CrossRef] [PubMed]

22. Schaller, F.; Sidelnikov, E.; Theiler, R.; Egli, A.; Staehelin, H.B.; Dick, W.; Dawson-Hughes, B.; Grob, D.; Platz, A.; Can, U.; et al. Mild to moderate cognitive impairment is a major risk factor for mortality and nursing home admission in the first year after hip fracture. Bone 2012, 51, 347-352. [CrossRef] [PubMed]

23. Fabian, E.; Gerstorfer, I.; Thaler, H.W.; Stundner, H.; Biswas, P.; Elmadfa, I. Nutritional supplementation affects postoperative oxidative stress and duration of hospitalization in patients with hip fracture. Wien. Klin. Wochenschr. 2011, 123, 88-93. [CrossRef] [PubMed]

24. Botella-Carretero, J.I.; Iglesias, B.; Balsa, J.A.; Arrieta, F.; Zamarrón, I.; Vázquez, C. Perioperative oral nutritional supplements in normally or mildly undernourished geriatric patients submitted to surgery for hip fracture: A randomized clinical trial. Clin. Nutr. 2010, 29, 574-579. [CrossRef] [PubMed]

25. Hoekstra, J.C.; Goosen, J.H.; de Wolf, G.S.; Verheyen, C.C. Effectiveness of multidisciplinary nutritional care on nutritional intake, nutritional status and quality of life in patients with hip fractures: A controlled prospective cohort study. Clin. Nutr. 2011, 30, 455-461. [CrossRef] [PubMed]

26. Drevet, S.; Bioteau, C.; Mazière, S.; Couturier, P.; Merloz, P.; Tonetti, J.; Gavazzi, G. Prevalence of protein-energy malnutrition in hospital patients over 75 years of age admitted for hip fracture. Orthop. Traumatol. Surg. Res. 2014, 100, 669-674. [CrossRef] [PubMed]

27. Goisser, S.; Schrader, E.; Singler, K.; Bertsch, T.; Gefeller, O.; Biber, R.; Bail, H.J.; Sieber, C.C.; Volkert, D. Malnutrition According to Mini Nutritional Assessment Is Associated With Severe Functional Impairment in Geriatric Patients Before and up to 6 Months After Hip Fracture. J. Am. Med. Dir. Assoc. 2015, 16, 661-667. [CrossRef] [PubMed]

28. Li, H.J.; Cheng, H.S.; Liang, J.; Wu, C.C.; Shyu, Y.I.L. Functional recovery of older people with hip fracture: Does malnutrition make a difference? J. Adv. Nurs. 2013, 69, 1691-1703. [CrossRef] [PubMed]

29. Wyers, C.E.; Reijven, P.L.M.; Evers, S.M.A.A.; Willems, P.C.; Heyligers, I.C.; Verburg, A.D.; Van Helden, S.; Dagnelie, P.C. Cost-effectiveness of nutritional intervention in elderly subjects after hip fracture. A randomized controlled trial. Osteoporos. Int. 2013, 24, 151-162. [CrossRef] [PubMed] 
30. Inoue, T.; Misu, S.; Tanaka, T.; Sakamoto, H.; Iwata, K.; Chuman, Y.; Ono, R. Pre-fracture nutritional status is predictive of functional status at discharge during the acute phase with hip fracture patients: A multicenter prospective cohort study. Clin. Nutr. 2017, 36, 1320-1325. [CrossRef] [PubMed]

31. Helminen, H.; Luukkaala, T.; Saarnio, J.; Nuotio, M. Comparison of the Mini-Nutritional Assessment short and long form and serum albumin as prognostic indicators of hip fracture outcomes. Injury 2017, 48, 903-908. [CrossRef] [PubMed]

32. Mazzola, P.; Ward, L.; Zazzetta, S.; Broggini, V.; Anzuini, A.; Valcarcel, B.; Brathwaite, J.S.; Pasinetti, G.M.; Bellelli, G.; Annoni, G. Association Between Preoperative Malnutrition and Postoperative Delirium After Hip Fracture Surgery in Older Adults. J. Am. Geriatr. Soc. 2017, 65, 1222-1228. [CrossRef] [PubMed]

33. Mansell, P.I.; Rawlings, J.; Allison, S.P.; Bendall, M.J.; Pearson, M.; Bassey, E.J.; Bastow, M. Low anthropometric indices in elderly females with fractured neck of femur. Clin. Nutr. 1990, 9, 190-194. [CrossRef]

34. Villani, A.M.; Miller, M.D.; Cameron, I.D.; Kurrle, S.; Whitehead, C.; Crotty, M. Development and relative validity of a new field instrument for detection of geriatric cachexia: Preliminary analysis in hip fracture patients. J. Cachexia. Sarcopenia Muscle 2013, 4, 209-216. [CrossRef] [PubMed]

35. Bell, J.J.; Bauer, J.D.; Capra, S.; Pulle, R.C. Concurrent and predictive evaluation of malnutrition diagnostic measures in hip fracture inpatients: A diagnostic accuracy study. Eur. J. Clin. Nutr. 2014, 68, 358-362. [CrossRef] [PubMed]

36. Espaulella, J.; Guyer, H.; Diaz-Escriu, F.; Mellado-Navas, J.A.; Castells, M.; Pladevall, M. Nutritional supplementation of elderly hip fracture patients. A randomized, double-blind, placebo-controlled trial. Age Ageing 2000, 29, 425-431. [CrossRef] [PubMed]

37. Baumgarten, M.; Margolis, D.J.; Orwig, D.L.; Shardell, M.D.; Hawkes, W.G.; Langenberg, P.; Palmer, M.H.; Jones, P.S.; McArdle, P.F.; Sterling, R.; et al. Pressure Ulcers in Elderly Patients with Hip Fracture Across the Continuum of Care. J. Am. Geriatr. Soc. 2009, 57, 863-870. [CrossRef] [PubMed]

38. Penrod, J.D.; Litke, A.; Hawkes, W.G.; Magaziner, J.; Koval, K.J.; Doucette, J.T.; Silberzweig, S.B.; Siu, A.L. Heterogeneity in hip fracture patients: Age, functional status, and comorbidity. J. Am. Geriatr. Soc. 2007, 55, 407-413. [CrossRef] [PubMed]

39. Hommel, A.; Bjorkelund, K.B.; Thorngren, K.-G.; Ulander, K. Nutritional status among patients with hip fracture in relation to pressure ulcers. Clin. Nutr. 2007, 26, 589-596. [CrossRef] [PubMed]

40. Cabrerizo, S.; Cuadras, D.; Gomez-Busto, F.; Artaza-Artabe, I.; Marin-Ciancas, F.; Malafarina, V. Serum albumin and health in older people: Review and meta analysis. Maturitas 2015, 81, 17-27. [CrossRef] [PubMed]

41. Formiga, F.; Chivite, D.; Mascaró, J.; Ramón, J.M.; Pujol, R. No correlation between mini-nutritional assessment (short form) scale and clinical outcomes in 73 elderly patients admitted for hip fracture. Aging Clin. Exp. Res. 2005, 17, 343-346. [CrossRef] [PubMed]

42. Perez-Barquero, M.M. Desnutricion como factor pronostico en ancianos con fractura de cadera. Med. Clin. 2007, 128, 721-728. [CrossRef]

43. Miyanishi, K.; Jingushi, S.; Torisu, T. Mortality after hip fracture in Japan: The role of nutritional status. J. Orthop. Surg. 2010, 18, 265-270. [CrossRef] [PubMed]

44. Flodin, L.; Laurin, A.; Lökk, J.; Cederholm, T.; Hedström, M. Increased 1-year survival and discharge to independent living in overweight hip fracture patients. Acta Orthop. 2016, 87, 146-151. [CrossRef] [PubMed]

45. Cenzer, I.S.; Tang, V.; Boscardin, W.J.; Smith, A.K.; Ritchie, C.; Wallhagen, M.I.; Espaldon, R.; Covinsky, K.E. One-Year Mortality After Hip Fracture: Development and Validation of a Prognostic Index. J. Am. Geriatr. Soc. 2016, 64, 1863-1868. [CrossRef] [PubMed]

46. Gumieiro, D.N.; Rafacho, B.P.M.; Gonçalves, A.F.; Tanni, S.E.; Azevedo, P.S.; Sakane, D.T.; Carneiro, C.A.S.; Gaspardo, D.; Zornoff, L.A.M.; Pereira, G.J.C.; et al. Mini Nutritional Assessment predicts gait status and mortality 6 months after hip fracture. Br. J. Nutr. 2013, 109, 1657-1661. [CrossRef] [PubMed]

47. Uriz-Otano, F.; Pla-Vidal, J.; Tiberio-Lopez, G.; Malafarina, V. Factors associated to institutionalization and mortality over three years, in elderly people with a hip fracture-An observational study. Maturitas 2016, 89, 9-15. [CrossRef] [PubMed]

48. Sullivan, D.H.; Nelson, C.L.; Klimberg, V.S.; Bopp, M.M. Nightly enteral nutrition support of elderly hip fracture patients: A pilot study. J. Am. Coll. Nutr. 2004, 23, 683-691. [CrossRef] [PubMed] 
49. Duncan, D.G.; Beck, S.J.; Hood, K.; Johansen, A. Using dietetic assistants to improve the outcome of hip fracture: A randomised controlled trial of nutritional support in an acute trauma ward. Age Ageing 2006, 35, 148-153. [CrossRef] [PubMed]

50. Schurch, M.-A. Protein Supplements Increase Serum Insulin-Like Growth Factor-I Levels and Attenuate Proximal Femur Bone Loss in Patients with Recent Hip Fracture. Ann. Intern. Med. 1998, 128, 801. [CrossRef] [PubMed]

51. Bruce, D.; Laurance, I.; McGuiness, M.; Ridley, M.; Goldswain, P. Nutritional supplements after hip fracture: Poor compliance limits effectiveness. Clin. Nutr. 2003, 22, 497-500. [CrossRef]

52. Myint, M.W.; Wu, J.; Wong, E.; Chan, S.P.; To, T.S.; Chau, M.W.; Ting, K.H.; Fung, P.M.; Au, K.S. Clinical benefits of oral nutritional supplementation for elderly hip fracture patients: A single blind randomised controlled trial. Age Ageing 2013, 42, 39-45. [CrossRef] [PubMed]

53. Tidermark, J.; Ponzer, S.; Carlsson, P.; Soderqvist, A.; Brismar, K.; Tengstrand, B.; Cederholm, T. Effects of protein-rich supplementation and nandrolone in lean elderly women with femoral neck fractures. Clin. Nutr. 2004, 23, 587-596. [CrossRef] [PubMed]

54. Malafarina, V.; Uriz-Otano, F.; Malafarina, C.; Martinez, J.A.; Zulet, M.A. Effectiveness of nutritional supplementation on sarcopenia and recovery in hip fracture patients. A multi-centre randomized trial. Maturitas 2017, 101, 42-50. [CrossRef] [PubMed]

55. Ekinci, O.; Yanik, S.; Terzioglu Bebitoglu, B.; Yilmaz Akyuz, E.; Dokuyucu, A.; Erdem, S. Effect of Calcium beta-Hydroxy-beta-Methylbutyrate (CaHMB), Vitamin D, and Protein Supplementation on Postoperative Immobilization in Malnourished Older Adult Patients With Hip Fracture: A Randomized Controlled Study. Nutr. Clin. Pract. 2016. [CrossRef] [PubMed]

56. Houwing, R. A randomised, double-blind assessment of the effect of nutritional supplementation on the prevention of pressure ulcers in hip-fracture patients. Clin. Nutr. 2003, 22, 401-405. [CrossRef]

57. Agarwal, E.; Miller, M.; Yaxley, A.; Isenring, E. Malnutrition in the elderly: A narrative review. Maturitas 2013, 76, 296-302. [CrossRef] [PubMed]

58. Camina-Martin, M.A.; de Mateo-Silleras, B.; Malafarina, V.; Lopez-Mongil, R.; Nino-Martin, V.; Lopez-Trigo, J.A.; Redondo-del-Rio, M.P. Nutritional status assessment in geriatrics: Consensus declaration by the Spanish Society of Geriatrics and Gerontology Nutrition Work Group. Maturitas 2015, 81, 414-419. [CrossRef] [PubMed]

59. Granic, A.; Mendonça, N.; Hill, T.; Jagger, C.; Stevenson, E.; Mathers, J.; Sayer, A. Nutrition in the Very Old. Nutrients 2018, 10, 269. [CrossRef] [PubMed]

60. Watterson, C.; Fraser, A.; Banks, M.; Isenring, E.; Miller, M.; Silvester, C.; Hoevenaars, R.; Bauer, J.; Vivanti, A.; Ferguson, M. Evidence based practice guidelines for the nutritional management of malnutrition in adult patients across the continuum of care. Nutr. Diet. 2009, 66, S1-S34. [CrossRef]

61. Leggo, M.; Banks, M.; Isenring, E.; Stewart, L.; Tweeddale, M. A quality improvement nutrition screening and intervention program available to Home and Community Care eligible clients. Nutr. Diet. 2008, 65, 162-167. [CrossRef]

62. García Lázaro, M.; Montero Pérez-Barquero, M.; Carpintero Benítez, P. Importancia de la malnutrición y otros factores médicos en la evolución de los pacientes con fractura de cadera. An. Med. Interna 2004, 21, 557-563. [CrossRef] [PubMed]

63. Malafarina, V.; Uriz-Otano, F.; Gil-Guerrero, L.; Iniesta, R. The anorexia of ageing: Physiopathology, prevalence, associated comorbidity and mortality. A systematic review. Maturitas 2013, 74, $293-302$. [CrossRef] [PubMed]

64. De Laet, C.; Kanis, J.A.; Oden, A.; Johanson, H.; Johnell, O.; Delmas, P.; Eisman, J.A.; Kroger, H.; Fujiwara, S.; Garnero, P.; et al. Body mass index as a predictor of fracture risk: A meta-analysis. Osteoporos. Int. 2005, 16, 1330-1338. [CrossRef] [PubMed]

65. Malafarina, V.; Úriz-Otano, F.; Iniesta, R.; Gil-Guerrero, L. Sarcopenia in the elderly: Diagnosis, physiopathology and treatment. Maturitas 2012, 71. [CrossRef] [PubMed]

66. Ben-Yacov, L.; Ainembabazi, P.; Stark, A.H. Is it time to update body mass index standards in the elderly or embrace measurements of body composition? Eur. J. Clin. Nutr. 2017, 71, 1029-1032. [CrossRef] [PubMed]

67. Rolland, Y.; Gallini, A.; Cristini, C.; Schott, A.; Blain, H.; Beauchet, O.; Cesari, M. Body-composition predictors of mortality in women aged $\$ 75 \mathrm{y}$ : Data from a large population-based cohort study with a 17-y follow-up 1-4. Am. J. Clin. Nutr. 2014, 1352-1361. [CrossRef] [PubMed] 
68. Graf, C.E.; Karsegard, V.L.; Spoerri, A.; Makhlouf, A.M.; Ho, S.; Herrmann, F.R.; Genton, L. Body composition and all-cause mortality in subjects older than 65 y. Am. J. Clin. Nutr. 2015, 101, 760-767. [CrossRef] [PubMed]

69. Iwata, M.; Kuzuya, M.; Kitagawa, Y.; Iguchi, A. Prognostic value of serum albumin combined with serum C-reactive protein levels in older hospitalized patients: Continuing importance of serum albumin. Aging Clin. Exp. Res. 2006, 18, 307-311. [CrossRef] [PubMed]

70. Drescher, T.; Singler, K.; Ulrich, A.; Koller, M.; Keller, U.; Christ-Crain, M.; Kressig, R.W. Comparison of two malnutrition risk screening methods (MNA and NRS 2002) and their association with markers of protein malnutrition in geriatric hospitalized patients. Eur. J. Clin. Nutr. 2010, 64, 887-893. [CrossRef] [PubMed]

71. Brooke, J.; Ojo, O. Enteral nutrition in dementia: A systematic review. Nutrients 2015, 7, 2456-2468. [CrossRef] [PubMed]

72. Portegijs, E.; Buurman, B.M.; Essink-Bot, M.L.; Zwinderman, A.H.; de Rooij, S.E. Failure to Regain Function at 3 months After Acute Hospital Admission Predicts Institutionalization Within 12 Months in Older Patients. J. Am. Med. Dir. Assoc. 2012, 13, 569.e1-569.e7. [CrossRef] [PubMed]

73. Ethgen, O.; Hiligsmann, M.; Burlet, N.; Reginster, J.Y. Cost-effectiveness of personalized supplementation with vitamin D-rich dairy products in the prevention of osteoporotic fractures. Osteoporos. Int. 2016, 27, 301-308. [CrossRef] [PubMed]

74. Rolland, Y.; Pillard, F.; Lauwers-Cances, V.; Busquere, F.; Vellas, B.; Lafont, C. Rehabilitation outcome of elderly patients with hip fracture and cognitive impairment. Disabil. Rehabil. 2004, 26, 425-431. [CrossRef] [PubMed]

75. Huang, S.-W.; Lin, J.-W.; Liou, T.-H.; Lin, H.-W. Cohort study evaluating the risk of hip fracture among patients with dementia in Taiwan. Int. J. Geriatr. Psychiatry 2015, 30, 695-701. [CrossRef] [PubMed]

76. Penrod, J.D.; Litke, A.; Hawkes, W.G.; Magaziner, J.; Doucette, J.T.; Koval, K.J.; Silberzweig, S.B.; Egol, K.A.; Siu, A.L. The association of race, gender, and comorbidity with mortality and function after hip fracture. J. Gerontol. Ser. A Biol. Sci. Med. Sci. 2008, 63, 867-872. [CrossRef]

77. Karampampa, K.; Ahlbom, A.; Michaëlsson, K.; Andersson, T.; Drefahl, S.; Modig, K. Declining incidence trends for hip fractures have not been accompanied by improvements in lifetime risk or post-fracture survival-A nationwide study of the Swedish population 60 years and older. Bone 2015, 78, 55-61. [CrossRef] [PubMed]

78. Rodríguez-Pascual, C.; Vilches-Moraga, A.; Paredes-Galán, E.; Ferrero-Marinez, A.I.; Torrente-Carballido, M.; Rodríguez-Artalejo, F. Comprehensive geriatric assessment and hospital mortality among older adults with decompensated heart failure. Am. Heart J. 2012, 164, 756-762. [CrossRef] [PubMed]

79. Pérez-Ros, P.; Martinez-Arnau, F.M.; Malafarina, V.; Tarazona-Santabalbina, F.J. A one-year proprioceptive exercise programme reduces the incidence of falls in community-dwelling elderly people: A before-after non-randomised intervention study. Maturitas 2016, 94, 155-160. [CrossRef] [PubMed]

80. Bajaj, N.S.; Bhatia, V.; Sanam, K.; Ather, S.; Hashim, T.; Morgan, C.; Fonarow, G.C.; Nanda, N.C.; Prabhu, S.D.; Adamopoulos, C.; et al. Impact of atrial fibrillation and heart failure, independent of each other and in combination, on mortality in community-dwelling older adults. Am. J. Cardiol. 2015, 114, 909-913. [CrossRef] [PubMed]

81. Butrous, H.; Hummel, S.L. Heart Failure in Older Adults. Can. J. Cardiol. 2016, 32, 1140-1147. [CrossRef] [PubMed]

82. Foss, N.B.; Kehlet, H. Mortality analysis in hip fracture patients: Implications for design of future outcome trials. Br. J. Anaesth. 2005, 94, 24-29. [CrossRef] [PubMed]

83. Volkert, D.; Berner, Y.N.; Berry, E.; Cederholm, T.; Bertrand, P.C.; Milne, A.; Palmblad, J.; Schneider, S.; Sobotka, L.; Stanga, Z.; et al. ESPEN guidelines on enteral nutrition: Geriatrics. Clin. Nutr. 2006, 25, 330-360. [CrossRef] [PubMed]

(C) 2018 by the authors. Licensee MDPI, Basel, Switzerland. This article is an open access article distributed under the terms and conditions of the Creative Commons Attribution (CC BY) license (http:/ / creativecommons.org/licenses/by/4.0/). 